\title{
Pitchfork and octopus bifurcations in a hyperelastic tube subjected to compression: Analytical post-bifurcation solutions and imperfection sensitivity
}

Mathematics and Mechanics of Solids 20I5, Vol. 20(I) 25-52 (C) The Author(s) 2014 Reprints and permissions: sagepub.co.uk/journalsPermissions.nav DOI: $10.1|77 / 108| 2865 \mid 4543597$ mms.sagepub.com

\section{Hui-Hui Dai}

Department of Mathematics, City University of Hong Kong, Hong Kong, China

\author{
Fan-Fan Wang \\ Department of Mathematics, East China University of Science and Technology, Shanghai, \\ China
}

\author{
Jiong Wang \\ Chair of Applied Mechanics, University of Erlangen-Nuremberg, Erlangen, Germany
}

\author{
Jian Xu \\ School of Aerospace Engineering and Applied Mechanics, Tongji University, Shanghai, China
}

Received 29 August 2013; accepted 29 October 2013

\begin{abstract}
This paper studies the axisymmetric deformations of a nonlinearly hyperelastic tube subjected to axial compression. We aim at investigating the critical buckling stresses and modes, deriving the analytical solutions for the post-bifurcation deformations and studying the imperfection sensitivity. For a general isotropic hyperelastic tube, a coupled seriesasymptotic method is utilized to derive two simplified model equations with specified constraints on the tube geometry. Then, we specialize to the Blatz-Ko material. With greased end conditions, through linear bifurcation analysis, we obtain the critical stress values and the corresponding mode numbers. The analytical solutions for the post-bifurcation states are constructed by the multiple scales method. By examining the solution behavior in the post-bifurcation regime, it is found that a thick tube could be considerably softer than a thin one. The singularities theory is used to consider the imperfection sensitivity, which reveals the mechanism is the existence of two modes corresponding to the same critical stress. Numerical solutions are also obtained which confirm the validity of the analytical solutions.
\end{abstract}

\section{Keywords}

Tube, post-bifurcation solutions, hyperelasticity, asymptotic analysis, imperfection sensitivity

Dedicated to Professor KR Rajagopal.

Corresponding author:

Hui-Hui Dai, Department of Mathematics, City University of Hong Kong, 83 Tat Chee Avenue, Hong Kong, China.

Email: mahhdai@cityu.edu.hk 


\section{Introduction}

Buckling of elastic shell structures has been studied extensively. For example, Koiter [1] (see also [2]) developed an asymptotic approach for studying the post-bifurcation behavior and imperfection sensitivity of an elastic cylindrical shell under compression. Hutchinson [3] conducted a post-bifurcation analysis for externally pressurized spherical shells. Hunt et al. [4] used the von Karman equations of shells to characterize localization and periodicity in cylindrical shells. Usually, in most analytical studies, only geometrical nonlinearity is taken into account. Relatively speaking, few works deal with the postbifurcation behavior of shell structures within the framework of finite elasticity, which takes into account both geometrical and material nonlinearity. One major reason is probably the complexity of the governing field equations. As mentioned in a review article [5], "the equations of motion...for an isotropic hyperelastic solid comprise a formidable system of nonlinear, partial differential equations (PDEs). The complexity of this system and its potential for generating non-unique solutions for even the simplest boundary-value problems,..., overwhelm our ability to solve them generally.' As a consequence, most theoretical studies on hyperelastic cylindrical shells in literature were carried out within the framework of linearized theories. By assuming the disturbances superimposed on a finite deformation are small, governing PDEs can be linearized. Then, it is possible to deduce the bifurcation modes and critical loads.

The first theoretical study on the bifurcation of an incompressible hyperelastic thick-walled cylindrical tube was given in [6]. More than forty years later, this problem was revisited in [7-9] and some new results for both axisymmetric and asymmetric buckling instabilities were obtained. Based on the threedimensional incremental equations for both thin-walled and thick-walled tubes in [10, 11], numerical studies were carried out in [12] on deformations of a thick-walled tube under axial loading and external pressure. For a compressible hyperelastic tube under compression, a linear bifurcation analysis was carried out in [13]. More recently, by using the Stroh formalism, Goriely et al. [14] studied the compression of a tube composed of an incompressible hyperelastic material. Both buckling modes and barreling modes were considered by them. In particular, they found the values of the geometric parameters at which there is a transition between buckling and barreling.

While linear bifurcation analysis can provide many insights, it cannot provide information on the post-bifurcation behavior (e.g. the answer for the very basic question of the bifurcation type). Actually, it was mentioned in [14]: 'It is also enticing to consider the possibility of performing an analytical postbuckling analysis of the solutions ... We leave this daunting task for another day.' In this paper, we shall conduct an analytical study on the axisymmetrical post-bifurcation states of a compressible hyperelastic tube under compression.

It is well known that it is difficult to obtain exact solutions for post-bifurcation states from the field equations. Usually, one has to resort to the approximate analytical or numerical solutions. Recently, a methodology of coupled series-asymptotic expansions, which was first developed to study nonlinear elastic waves (see $[15,16])$, has been successfully used in constructing approximate analytical solutions within the framework of field equations for thin/slender structures composed of hyperelastic materials (see [17-21]). In this paper, this methodology will be adopted to study the axisymmetric deformations of a compressible hyperelastic tube subjected to uniaxial compression. One of our motivations is to obtain the analytical post-bifurcation solutions, which have not been obtained before in the current setting, to the best of the authors' knowledge. As we shall see, the analytical results reveal many insights. For example, for the single-mode case it is found that the bifurcation is of the supercritical pitchfork bifurcations type. For the case of two modes corresponding to one critical stress, there are eight bifurcated branches (four are supercritical and four are subcritical), which is called an octopus bifurcation. It appears that such a bifurcation has not been reported before in the context of cylindrical shells. Another important feature of the present work is that the post-bifurcation solutions are deduced from the field equations without ad hoc assumptions, which are often used in literature on postbuckling analysis.

An important issue in shell structures is imperfection sensitivity. With the explicit amplitude equations, it becomes possible to use the singularities theory to study imperfection sensitivity (see [22, 23]). One of the important advantages of this analytical approach (it appears that it has not been widely adopted in engineering literature) is that the qualitative information can be deduced without the need to make assumptions about the particular form of the imperfection (which is often done in the asymptotic 
approach for studying imperfection sensitivity). The analysis reveals that the mechanism for imperfection sensitivity is that there are two modes corresponding to a critical load. It is then deduced that usually a thin tube is imperfection-sensitive while in general a thick tube is not, which is in agreement with some experimental observations as mentioned in [24].

Experiments on the compression of tubes have also been extensively carried out. One of the interesting experiments was done by Willis [25] (see also [5] for a description). Motivated by this experimental work, Dorfmann and Haughton [13] studied the incremental equations for a compressible hyperelastic cylindrical tube compressed at two frictionless ends. It is worth mentioning that a number of experiments on compression of plastic cylindrical tubes has been carried out (see [24, 26, 27]). The present study is carried out within the framework of three-dimensional field equations of finite elasticity without an assumption about the form of the displacement field. Since a plastic material may be regarded as a nonlinear elastic one in a pure loading process, the results in this paper may also provide some qualitative information on the compression of plastic tubes. We also notice that the first bifurcation mode is axisymmetric for not very thin tubes according to the experiments in [26] (for diameter-to-thickness ratios of 20,40,59.7) and [24] (for diameter-to-thickness ratios between 23 and 52). This is one reason why we study axisymmetric deformations in this paper.

Cylindrical tubes are commonly used as energy absorbers due to their high stiffness and strength combined with low weight when subjected to axial load (see [28]). Metallic cylindrical tubes in automobiles can absorb the kinetic crash energy to limit the damage in a crash. The capability of absorbing energy depends on the type of buckling mode, which is related to the geometry of the tube and the boundary conditions. In this paper, another motivation is to study the effect of the geometry of the tube on the post-bifurcation states. With analytical solutions, this effect can be examined easily. For energy absorption purposes, one needs the axial stress to be small in the postbifurcation regime. Usually, people would think that a thin tube is softer than a thick one. While this is true up to the bifurcation, we find that in the post-bifurcation regime a thick tube could be softer than a thin tube.

The structure of this paper is organized as follows. In Section 2, we set up the three-dimensional axisymmetric field equations and the traction-free boundary conditions for a general hyperelastic material. In Section 3, we derive two coupled nonlinear ordinary differential equations (ODEs) by the combined series-asymptotic expansions method. In Section 4, the tube is further assumed to be composed of a Blatz-Ko material. In Section 5, with sliding or greased conditions at two ends, linear bifurcation analysis is made to obtain the critical stress values and the corresponding mode numbers. The influences of the geometrical parameters are also examined in detail in this section. In Section 6, we derive the approximate analytical solutions for the post-bifurcation states. It turns out that this nonlinear bifurcation analysis can recover the critical stress values and the mode numbers. By using the singularities theory, in Section 7 the imperfection sensitivity is analyzed. In Section 8, numerical solutions are compared with the approximate analytical solutions and very good agreement is found. Finally, some concluding remarks are presented.

\section{Field equations}

We consider the axisymmetric deformation of a three-dimensional hyperelastic cylindrical tube subjected to a static axial force at one end with zero axial displacement at the other end. The inner and outer surfaces of the tube are traction-free and the end boundary conditions will be considered later. In the stressfree configuration, the inner and outer radii of the tube are, respectively, $a$ and $b$, and the length is $l$. We take the cylindrical polar coordinates system and denote by $(R, \Theta, Z)$ and $(r, \theta, z)$ the coordinates of a material point of the tube in the reference and current configurations, respectively. The displacements of a material point can be represented as

$$
U(R, Z)=r(R, Z)-R, \quad \Theta=\theta, \quad W(R, Z)=z(R, Z)-Z .
$$

We introduce the orthonormal bases associated with the cylindrical coordinates and denote these by $\left\{\mathbf{E}_{R}, \mathbf{E}_{\Theta}, \mathbf{E}_{Z}\right\}$ and $\left\{\mathbf{e}_{r}, \mathbf{e}_{\theta}, \mathbf{e}_{z}\right\}$ in the reference and current configurations, respectively. Then the deformation gradient tensor $\mathbf{F}$ with respect to the bases $\left\{\mathbf{e}_{i} \otimes \mathbf{E}_{j}\right\}$ is given by 


$$
\mathbf{F}=\left(\begin{array}{ccc}
1+U_{R} & 0 & U_{Z} \\
0 & 1+\frac{U}{R} & 0 \\
W_{R} & 0 & 1+W_{Z}
\end{array}\right)
$$

For an isotropic hyperelastic material, the strain-energy function $\Phi$ is a function of the three principle stretches $e_{1}, e_{2}$ and $e_{3}$ of the Lagrangian strain tensor $\mathbf{E}=(1 / 2)\left(\mathbf{F}^{\mathrm{T}} \mathbf{F}-\mathbf{I}\right)$, that is, $\Phi=\Phi\left(e_{1}, e_{2}, e_{3}\right)$, where $\mathbf{I}$ is the identity tensor. The conjugate stress tensor is given by

$$
\mathbf{T}=\frac{\partial \Phi}{\partial \mathbf{E}}
$$

with components $T_{i j}=\partial \Phi / \partial E_{j i}$. If the strains are small, it is possible to expand $T_{i j}$ in terms of the strains up to any order. The formula containing terms up to the third-order material nonlinearity is

$$
T_{i j}=A_{i j k l}^{(1)} E_{l k}+\frac{1}{2} A_{i j k l m n}^{(2)} E_{l k} E_{n m}+\frac{1}{6} A_{i j k l m n p q}^{(3)} E_{l k} E_{n m} E_{q p}+O\left(\left|E_{s t}\right|^{4}\right),
$$

where

$$
\begin{aligned}
& A_{i j k l}^{(1)}=\left.\frac{\partial^{2} \Phi}{\partial E_{j i} \partial E_{l k}}\right|_{\mathbf{E}=\mathbf{0}}, \quad A_{i j k l m n}^{(2)}=\left.\frac{\partial^{3} \Phi}{\partial E_{j i} \partial E_{l k} \partial E_{n m}}\right|_{\mathbf{E}=\mathbf{0}}, \\
& A_{i j k l m n p q}^{(3)}=\left.\frac{\partial^{4} \Phi}{\partial E_{j i} \partial E_{l k} \partial E_{n m} \partial E_{q p}}\right|_{\mathbf{E}=\mathbf{0}}
\end{aligned}
$$

are the incremental elastic moduli, which can be calculated once a specific form of the strain energy function is given. The formulae for $A_{i j k l}^{(1)}$ and $A_{i j k l m n}^{(2)}$ can be found in [29]. By a similar procedure, the formula for $A_{i j k l m n p q}^{(3)}$ can also be deduced. The non-zero elastic moduli are given in the Appendix. From the conjugate stress tensor $\mathbf{T}$, the nominal stress tensor $\mathbf{S}$ is given by $\mathbf{S}=\mathbf{T F}^{\mathrm{T}}$.

For a static equilibrium state of the tube (neglecting the body force), we have the following two field equations:

$$
\begin{gathered}
\frac{\partial S_{R r}}{\partial R}+\frac{\partial S_{Z r}}{\partial Z}+\frac{S_{R r}-S_{\Theta \theta}}{R}=0, \\
\frac{\partial S_{R z}}{\partial R}+\frac{\partial S_{Z z}}{\partial Z}+\frac{S_{R z}}{R}=0 .
\end{gathered}
$$

We consider the case where the inner and outer surfaces of the tube are traction-free. Thus, we have the traction-free boundary conditions

$$
S_{R r}=0, \quad S_{R z}=0, \quad \text { at } \quad R=a, b .
$$

Equations (2.6a) and (2.6b) together with (2.7) provide the governing equations and the boundary conditions for two unknowns $U$ and $W$, which compose a complicated coupled nonlinear system of PDEs. It is almost formidable to construct exact solutions from those PDEs for the postbifurcation states. The aim is to construct explicit approximate analytical solutions (which is also a very difficult task). For this purpose, we shall adopt an approach involving combined series-asymptotic expansions to tackle this complicated problem. A similar methodology has been utilized to study nonlinear elastic waves and phase transitions in incompressible materials (see $[15,16,30])$. In the following section, we shall first nondimensionalize the system to identify relevant small variables and small parameters. Then we shall derive the reduced model equations by this method of coupled series-asymptotic expansions. 


\section{Nondimensionalization and series-asymptotic reductions}

According to [15], for this kind of axisymmetric problem it is convenient to introduce the transformation

$$
U=R u, \quad s=R^{2} .
$$

Equation (3.1) 1 is introduced based on the observation that $U=R \lambda$ ( $\lambda$ is a constant) for a homogeneous deformation induced by axial compression, while $(3.1)_{2}$ is introduced to eliminate $1 / R$ in $(2.6 \mathrm{a})$ and (2.6b). The length $l$ of the tube is a natural choice to scale the spatial variables. To be consistent with (2.4), we consider the case where under compression one end of the tube moves a distance up to $h$ with the constraint of $\varepsilon=h / l$ being small ( $h$ is then a measure of the axial displacement). Thus, we introduce the following scalings:

$$
s=l^{2} \tilde{s}, \quad Z=l \tilde{z}, \quad u=\frac{h}{l} \tilde{u}, \quad W=h \tilde{w} .
$$

After substituting (3.2) into (2.6a), (2.6b) and (2.7), we obtain a dimensionless system, in which two geometric parameters $\nu_{0}=a^{2} / l^{2}$ and $\nu_{1}=b^{2} / l^{2}$ arise. To use expansion methods to proceed forward, some restrictions on the geometric parameters have to be imposed. We consider the case where $\delta=\nu_{1}-\nu_{0}=(b+a)(b-a) / l^{2}$ is small such that $O\left(\delta^{2}\right)$ terms can be omitted. We point out that this implies a small thickness-to-length ratio, but the thickness-to-radius ratio does not need to be small at this stage.

For convenience of notation, we hereafter drop the tildes (i.e. $s$ means $\tilde{s}$ ). We note that since $0 \leq s-$ $\nu_{0} \leq \delta, s-\nu_{0}$ is a small variable. If we assume $u(s, z)$ and $w(s, z)$ are sufficiently smooth in $s$, we can take the following series expansions of $u$ and $w$ in the neighborhood of $s=\nu_{0}$ :

$$
\left\{\begin{array}{l}
u(s, z)=u_{0}(z)+\left(s-\nu_{0}\right) u_{1}(z)+\left(s-\nu_{0}\right)^{2} u_{2}(z)+\left(s-\nu_{0}\right)^{3} u_{3}(z)+\ldots \\
w(s, z)=w_{0}(z)+\left(s-\nu_{0}\right) w_{1}(z)+\left(s-\nu_{0}\right)^{2} w_{2}(z)+\left(s-\nu_{0}\right)^{3} w_{3}(z)+\ldots
\end{array}\right.
$$

Remark: Since $R / l-\sqrt{\nu_{0}}=\sqrt{\nu_{0}+s-\nu_{0}}-\sqrt{\nu_{0}}$, which can be expanded as a power series of $s-\nu_{0}$ for $\delta / \nu_{0}<1$, there is no need for the above expansions to contain $\left(R / l-\sqrt{\nu_{0}}\right)^{n}(n=1,2, \ldots)$ terms.

Substituting (3.3) into the nondimensionalized traction-free boundary conditions (2.7), we obtain the following four equations:

$$
\begin{aligned}
\nu_{0} \mathscr{D}_{1}\left(\mathbf{v}_{\mathbf{0}}\right) u_{1}+\varepsilon \nu_{0} \mathscr{D}_{2}\left(\mathbf{v}_{\mathbf{0}}\right) w_{1}+\left(\zeta_{1}+\zeta_{2}\right) u_{0}+\zeta_{2} w_{0 z}+\varepsilon^{2} \nu_{0} \mathscr{D}_{3}\left(\mathbf{v}_{\mathbf{0}}\right) u_{1}^{2}+\varepsilon \nu_{0} \mathscr{D}_{4}\left(\mathbf{v}_{\mathbf{0}}\right) u_{1} w_{1} \\
+\varepsilon \nu_{0} \mathscr{D}_{5}\left(\mathbf{v}_{\mathbf{0}}\right) w_{1}^{2}+\varepsilon^{2} \nu_{0}^{3}\left(4 \zeta_{1}+8 \eta_{1}+\frac{4}{3} \kappa_{1}\right) u_{1}^{3}+\varepsilon^{2} \nu_{0}^{2} 4\left(\zeta_{1}+\eta_{1}+\eta_{4}+\kappa_{5}\right) u_{1} w_{1}^{2}+\varepsilon \mathscr{D}_{6}\left(\mathbf{v}_{\mathbf{0}}\right) \\
+\varepsilon^{2} \mathscr{D}_{7}\left(\mathbf{v}_{\mathbf{0}}\right)=0 \\
\varepsilon \nu_{0} \mathscr{D}_{8}\left(\mathbf{v}_{\mathbf{0}}\right) u_{1}+\mathscr{D}_{9}\left(\mathbf{v}_{\mathbf{0}}\right) w_{1}+\zeta_{3} u_{0 z}+\varepsilon \nu_{0} \mathscr{D}_{10}\left(\mathbf{v}_{\mathbf{0}}\right) u_{1}^{2}+\varepsilon \nu_{0} \mathscr{D}_{11}\left(\mathbf{v}_{\mathbf{0}}\right) u_{1} w_{1}+\varepsilon \nu_{0} \mathscr{D}_{12}\left(\mathbf{v}_{\mathbf{0}}\right) w_{1}^{2} \\
+\varepsilon^{2} \nu_{0}^{2} 4\left(\zeta_{1}+\eta_{1}+\eta_{4}+\kappa_{5}\right) u_{1}^{2} w_{1}+\varepsilon^{2} \nu_{0}\left(4 \zeta_{1}+8 \eta_{4}+\frac{4}{3} \kappa_{9}\right) w_{1}^{3}+\varepsilon \mathscr{D}_{13}\left(\mathbf{v}_{\mathbf{0}}\right)+\varepsilon^{2} \mathscr{D}_{14}\left(\mathbf{v}_{\mathbf{0}}\right)=0, \\
\left(\left(3 \zeta_{1}+\zeta_{2}\right) u_{1}+4 \zeta_{1} \nu_{0} u_{2}+\zeta_{2} w_{1 z}\right)+\varepsilon \mathscr{D}_{15}\left(\mathbf{v}_{\mathbf{2}}\right)+\varepsilon^{2} \mathscr{D}_{16}\left(\mathbf{v}_{\mathbf{2}}\right)+\delta\left(\left(5 \zeta_{1}+\zeta_{2}\right) u_{2}\right. \\
\left.+6 \zeta_{1} \nu_{0} u_{3}+\zeta_{2} w_{2 z}+\varepsilon \mathscr{D}_{17}\left(\mathbf{v}_{\mathbf{3}}\right)+\varepsilon^{2} \mathscr{D}_{18}\left(\mathbf{v}_{\mathbf{3}}\right)\right)+O\left(\delta^{2}\right)=0, \\
\left(2 \zeta_{3} w_{1}+\zeta_{3} u_{0 z}+\nu_{0}\left(4 \zeta_{3} w_{2}+\zeta_{3} u_{1 z}\right)\right)+\varepsilon \mathscr{D}_{19}\left(\mathbf{v}_{\mathbf{2}}\right)+\varepsilon^{2} \mathscr{D}_{20}\left(\mathbf{v}_{\mathbf{2}}\right)+\delta\left(4 \zeta_{3} w_{2}+\zeta_{3} u_{1 z}\right. \\
\left.+\nu_{0}\left(6 \zeta_{3} w_{3}+\zeta_{3} u_{2 z}\right)+\varepsilon \mathscr{D}_{21}\left(\mathbf{v}_{\mathbf{3}}\right)+\varepsilon^{2} \mathscr{D}_{22}\left(\mathbf{v}_{\mathbf{3}}\right)\right)+O\left(\delta^{2}\right)=0,
\end{aligned}
$$

where $\mathbf{v}_{\mathbf{i}}(i=0,1,2,3)$ are the four vectors defined below: 


$$
\left\{\begin{array}{l}
\mathbf{v}_{\mathbf{0}}=\left(u_{0}, w_{0}\right), \mathbf{v}_{\mathbf{1}}=\left(u_{0}, u_{1}, w_{0}, w_{1}\right) \\
\mathbf{v}_{\mathbf{2}}=\left(u_{0}, u_{1}, u_{2}, w_{0}, w_{1}, w_{2}\right), \mathbf{v}_{\mathbf{3}}=\left(u_{0}, u_{1}, u_{2}, u_{3}, w_{0}, w_{1}, w_{2}, w_{3}\right)
\end{array}\right.
$$

$\eta_{j}, \zeta_{j}, \kappa_{j}(j=1, \ldots)$ are the elastic moduli given in the Appendix and $\mathscr{D}_{i}(i=1, \ldots, 22)$ are operators on the arguments whose long expressions are omitted. In obtaining (3.4c) and (3.4d), we have neglected terms higher than $O\left(\delta^{2}\right)$. In this system, there are eight unknowns $u_{i}$ and $w_{i}(i=0,1,2,3)$ if the abovementioned higher-order terms are omitted. The other four equations can be obtained from the field equations.

Substituting (3.3) into (2.6a) and (2.6b), the left-hand sides of the equations become two series in $s-\nu_{0}$ and all the coefficients of $\left(s-\nu_{0}\right)^{n}(n=0,1,2, \ldots)$ should be zero. From the coefficients of $\left(s-\nu_{0}\right)^{0}$ and $\left(s-\nu_{0}\right)^{1}$, four equations with the same eight unknowns are obtained:

$$
\begin{aligned}
& \nu_{0}\left(8 \zeta_{1} u_{1}+2\left(\zeta_{2}+\zeta_{3}\right) w_{1 z}+\zeta_{3} u_{0 z z}\right)+8 \zeta_{1} \nu_{0}^{2} u_{2}+\varepsilon \mathscr{D}_{23}\left(\mathbf{v}_{\mathbf{1}}\right)+\varepsilon^{2} \mathscr{D}_{24}\left(\mathbf{v}_{\mathbf{1}}\right)=0 \\
& 8 \zeta_{1} u_{1}+2\left(\zeta_{2}+\zeta_{3}\right) w_{1 z}+\zeta_{3} u_{0 z z}+\nu_{0}\left(32 \zeta_{1} u_{2}+4\left(\zeta_{2}+\zeta_{3}\right) w_{2 z}+\zeta_{3} u_{1 z z}\right) \\
& \quad+24 \zeta_{1} \nu_{0}^{2} u_{3}+\varepsilon \mathscr{D}_{25}\left(\mathbf{v}_{\mathbf{2}}\right)+\varepsilon^{2} \mathscr{D}_{26}\left(\mathbf{v}_{\mathbf{2}}\right)=0 \\
& 4 \zeta_{3} w_{1}+2\left(\zeta_{2}+\zeta_{3}\right) u_{0 z}+\zeta_{1} w_{0 z z}+\nu_{0}\left(8 \zeta_{3} w_{2}+2\left(\zeta_{2}+\zeta_{3}\right) u_{1 z}\right)+\varepsilon \mathscr{D}_{27}\left(\mathbf{v}_{\mathbf{1}}\right) \\
& \quad+\varepsilon^{2} \mathscr{D}_{28}\left(\mathbf{v}_{\mathbf{1}}\right)=0 \\
& 16 \zeta_{3} w_{2}+4\left(\zeta_{2}+\zeta_{3}\right) u_{1 z}+\zeta_{1} w_{1 z z}+\nu_{0}\left(24 \zeta_{3} w_{3}+4\left(\zeta_{2}+\zeta_{3}\right) u_{2 z}\right)+\varepsilon \mathscr{D}_{29}\left(\mathbf{v}_{\mathbf{2}}\right) \\
& \quad+\varepsilon^{2} \mathscr{D}_{30}\left(\mathbf{v}_{\mathbf{2}}\right)=0 .
\end{aligned}
$$

Now the governing system (2.6a) to (2.7) has been transformed into a closed system of eight equations (3.4) and (3.5) with eight unknowns $u_{i}$ and $w_{i}(i=0,1,2,3)$. A key that the present methodology works is that a closed finite system can be obtained!

We find that (3.4a) and (3.4b) are two algebraic equations for $u_{1}$ and $w_{1}$. By perturbation expansions in terms of the small parameter $\varepsilon$, we can obtain the asymptotic expressions of $u_{1}$ and $w_{1}$. Also, it can be seen that (3.5a) and (3.5c) are two linear algebraic equations for $u_{2}$ and $w_{2}$ and (3.5b) and (3.5d) are two linear algebraic equations for $u_{3}$ and $w_{3}$. Another key for the present methodology is that among the closed system of eight equations six are algebraic equations! As a result, $u_{i}$ and $w_{i}(i=1,2,3)$ can be expressed in terms of $u_{0}$ and $w_{0}$ (their long expressions are omitted here). Substituting these expressions into (3.4c) and (3.4d) and neglecting $O\left(\varepsilon^{3}, \varepsilon^{2} \nu_{0}, \delta \nu_{0},\left(\delta / \nu_{0}\right)^{2}\right)$ terms, we can obtain two equations with two unknowns $u_{0}$ and $w_{0}$. It turns out that one of the two equations can be integrated once. As a result, we have

$$
\begin{aligned}
& \frac{\zeta_{1}^{2}-\zeta_{2}^{2}}{2 \zeta_{1}} u_{0}+\frac{\left(\zeta_{1}-\zeta_{2}\right) \zeta_{2}}{2 \zeta_{1}} w_{0 z}+\varepsilon\left(a_{1} u_{0}^{2}+a_{2} u_{0} w_{0 z}+a_{3} w_{0 z}^{2}\right)+\varepsilon^{2}\left(a_{4} u_{0}^{3}+a_{5} u_{0}^{2} w_{0 z}\right. \\
& \left.+a_{6} u_{0} w_{0 z}^{2}+a_{7} w_{0 z}^{3}\right)+\varepsilon \nu_{0}\left(\frac{\zeta_{2}\left(-\zeta_{1}+\zeta_{2}\right)}{4 \zeta_{1}} u_{0 z}^{2}+\frac{\zeta_{2}\left(-\zeta_{1}+\zeta_{2}\right)}{2 \zeta_{1}} u_{0} u_{0 z z}+\frac{-\zeta_{1}^{2}+\zeta_{2}^{2}}{2 \zeta_{1}} u_{0 z z} w_{0 z}\right. \\
& \left.+\frac{-\zeta_{1}^{2}+\zeta_{2}^{2}}{2 \zeta_{1}} u_{0 z} w_{0 z z}\right)+\delta\left(\frac{\zeta_{1}^{2}-\zeta_{2}^{2}}{8 \zeta_{1}} w_{0 z z z}+\varepsilon\left(a_{8} u_{0 z}^{2}-\frac{\left(\zeta_{1}-\zeta_{2}\right)^{2}}{8 \zeta_{1}} u_{0} u_{0 z z}\right.\right. \\
& \left.+\frac{2 \zeta_{1}^{2}-\zeta_{1} \zeta_{2}-\zeta_{2}^{2}}{8 \zeta_{1}} u_{0 z z} w_{0 z}+a_{9} u_{0 z} w_{0 z z}+a_{10} w_{0 z z}^{2}+a_{11} u_{0} w_{0 z z z}+a_{12} w_{0 z} w_{0 z z z}\right) \\
& +\frac{1}{\nu_{0}}\left(\frac{-\zeta_{1}^{2}+\zeta_{2}^{2}}{2 \zeta_{1}} u_{0}+\frac{\zeta_{2}\left(-\zeta_{1}+\zeta_{2}\right)}{2 \zeta_{1}} w_{0 z}+\varepsilon\left(a_{13} u_{0}^{2}+a_{14} u_{0} w_{0 z}+a_{15} w_{0 z}^{2}\right)\right. \\
& \left.+\varepsilon^{2}\left(a_{16} u_{0}^{3}+a_{17} u_{0}^{2} w_{0 z}+a_{18} u_{0} w_{0 z}^{2}+a_{19} w_{0 z}^{3}\right)\right)=0
\end{aligned}
$$




$$
\begin{aligned}
A & +\frac{\zeta_{2}\left(-\zeta_{1}+\zeta_{2}\right)}{2 \zeta_{1}} u_{0}+\frac{-\zeta_{1}^{2}+\zeta_{2}^{2}}{2 \zeta_{1}} w_{0 z}+\varepsilon\left(a_{20} u_{0}^{2}+a_{21} u_{0} w_{0 z}+a_{22} w_{0 z}^{2}\right)+\varepsilon^{2}\left(a_{23} u_{0}^{3}\right. \\
& \left.+a_{24} u_{0}^{2} w_{0 z}+a_{25} u_{0} w_{0 z}^{2}+a_{26} w_{0 z}^{3}\right)+\varepsilon \nu_{0} \frac{-\zeta_{1}^{2}+\zeta_{2}^{2}}{4 \zeta_{1}} u_{0 z}^{2}+\delta\left(\frac{\zeta_{1}^{2}-\zeta_{2}^{2}}{8 \zeta_{1}} u_{0 z z}\right. \\
& +\varepsilon\left(\frac{\zeta_{2}\left(-\zeta_{1}+\zeta_{2}\right)}{16 \zeta_{1}} u_{0 z}^{2}+a_{34} u_{0} u_{0 z z}+a_{35} u_{0 z z} w_{0 z}+\frac{-\zeta_{1}^{2}+\zeta_{2}^{2}}{4 \zeta_{1}} u_{0 z} w_{0 z z}\right)+\frac{1}{\nu_{0}}\left(\varepsilon \left(a_{27} u_{0}^{2}\right.\right. \\
& \left.\left.\left.+a_{28} u_{0} w_{0 z}+a_{29} w_{0 z}^{2}\right)+\varepsilon^{2}\left(a_{30} u_{0}^{3}+a_{31} u_{0}^{2} w_{0 z}+a_{32} u_{0} w_{0 z}^{2}+a_{33} w_{0 z}^{3}\right)\right)\right)=0
\end{aligned}
$$

where $A$ is an integration constant and $a_{i}(i=1,2, \ldots, 35)$ are constants dependent on material constants. By considering the resultant force $T=\int_{0}^{2 \pi} \int_{a}^{b} S_{Z z} R d R d \Theta$ acting on a cross-section that is planar and perpendicular to the axial direction of the tube, we find that

$$
A=\frac{T}{2 \pi\left(b^{2}-a^{2}\right) \varepsilon}=\frac{\gamma\left(\zeta_{1}^{2}+\zeta_{1} \zeta_{2}-2 \zeta_{2}^{2}\right)}{2\left(\zeta_{1}+\zeta_{2}\right) \varepsilon},
$$

where $\gamma$ is the dimensionless engineering stress.

We point out that in deriving the above two asymptotic model equations $O\left(\varepsilon^{3}, \varepsilon^{2} \nu_{0}, \delta^{2}, \delta \nu_{0},\left(\delta / \nu_{0}\right)^{2}\right)$ terms have been neglected. For them to yield reliable results, we impose that these omitted terms should be less than $5.5 \%$. Now, we examine the restrictions in terms of the loading and geometrical parameters.

For $\delta^{2}<0.055,\left(\delta / \nu_{0}\right)^{2}<0.055$ and $\delta \nu_{0}<0.055$, we need, respectively, $(b / l)^{2}-(a / l)^{2}<0.2234, b / l$ $<1.106(a / l)$ and $\left((b / l)^{2}-(a / l)^{2}\right)(a / l)^{2}<0.055$. Also, suppose that we impose $\varepsilon<0.2$ ( $\varepsilon$ can be regarded as a loading parameter). Then, $\varepsilon^{3}<0.055$ automatically. Therefore, $\varepsilon^{2} \nu_{0}<0.055$ implies that $(a / l)<1.173$. Based on the above analysis, we plot the valid domain of $a / l$ and $b / l$ in Figure 1 .

We notice that within this domain we do not need the tube to be very thin (the diameter-to-thickness ratio can be as small as 20) or slender (the diameter-to-length ratio can be as large as 2.34). For most of the tubes used in the experiments by $[24,26,27]$ the geometrical parameters were in this domain.

\section{Governing equations for the Blatz-Ko material}

In Section 3, we obtained the governing equations for the axisymmetric deformations of a hyperelastic tube subjected to uniaxial compression. Once the strain-energy function of a specific hyperelastic material is given, we can calculate the corresponding elastic moduli through the formulae presented in the Appendix. Substituting the elastic moduli into the coefficients of (3.6a) and (3.6b), the model equations for the specific material can be determined.

In the following discussion, we shall take the Blatz-Ko material as an example. The strain-energy function of the Blatz-Ko material is

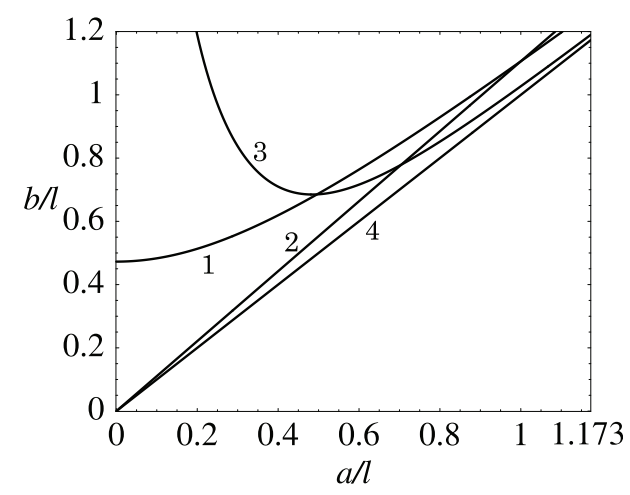

Figure I. The valid domain of $a / l$ and $b / l$ for the model equations is the one bounded by curves 2, 3 and 4 . Curves I, 2, 3 and 4 are determined by $(b / l)^{2}-(a / l)^{2}=0.2234, b / l=1.106(a / l),\left((b / l)^{2}-(a / l)^{2}\right)(a / l)^{2}=0.055$ and $b / l=a / l$, respectively. 


$$
\Phi\left(e_{1}, e_{2}, e_{3}\right)=\frac{\mu_{0}}{2}\left(\frac{1}{1+2 e_{1}}+\frac{1}{1+2 e_{2}}+\frac{1}{1+2 e_{3}}+2 \sqrt{\left(1+2 e_{1}\right)\left(1+2 e_{2}\right)\left(1+2 e_{3}\right)}-5\right),
$$

where $\mu_{0}$ is the shear modulus for infinitesimal deformations. By using the formulae in the Appendix, the non-zero elastic moduli (defined in (2.5)) of the Blatz-Ko material are

$$
\begin{aligned}
& \zeta_{1}=3 \mu_{0}, \quad \zeta_{2}=\mu_{0}, \quad \eta_{1}=-21 \mu_{0}, \quad \eta_{2}=-\mu_{0}, \quad \eta_{3}=\mu_{0}, \\
& \kappa_{1}=177 \mu_{0}, \quad \kappa_{2}=3 \mu_{0}, \quad \kappa_{3}=\mu_{0}, \quad \kappa_{4}=-\mu_{0} .
\end{aligned}
$$

Substituting (4.2) into (3.6a) and (3.6b) and retaining the original dimensional variables by letting $U$ $=\varepsilon u_{0}\left(a U\right.$ represents the radial displacement of a material point on the inner surface) and $G=\varepsilon w_{0 z}$ (which represents the axial strain of a material point on the inner surface), we can obtain the following governing equations for the Blatz-Ko material:

$$
\begin{aligned}
\frac{4}{3} U & -\frac{26}{9} U^{2}+\frac{398}{81} U^{3}+\left(\frac{1}{3}-\frac{1}{9} G+\frac{2}{27} U^{2}\right) G+\left(-\frac{1}{18}+\frac{1}{54} U\right) G^{2}+\frac{2}{81} G^{3} \\
& +\nu_{0}\left(-\frac{1}{6} U_{Z}^{2}-\frac{1}{3} U U_{Z Z}-\frac{4}{3} U_{Z Z} G-\frac{4}{3} U_{Z} G_{Z}\right)+\delta\left(\frac{7}{12} U_{Z Z} G-\frac{19}{36} U_{Z}^{2}-\frac{1}{6} U U_{Z Z}\right. \\
& +\frac{19}{36} U_{Z} G_{Z}-\frac{16}{9} G_{Z}^{2}+\left(\frac{1}{3}-\frac{1}{9} U-\frac{16}{9} G\right) G_{Z Z}+\frac{1}{\nu_{0}}\left(-\frac{4}{3} U+\frac{34}{9} U^{2}-\frac{218}{27} U^{3}\right. \\
& \left.\left.+\left(-\frac{1}{3}+\frac{5}{9} U-\frac{4}{3} U^{2}\right) G+\left(\frac{1}{9}-\frac{1}{3} U\right) G^{2}-\frac{2}{27} G^{3}\right)\right)=0, \\
\frac{5}{4} \gamma & -\frac{1}{3} U+\frac{1}{18} U^{2}-\frac{2}{81} U^{3}+\left(-\frac{4}{3}+\frac{1}{9} U-\frac{1}{54} U^{2}\right) G+\left(\frac{26}{9}-\frac{2}{27} U\right) G^{2}-\frac{398}{81} G^{3} \\
& -\frac{2 \nu_{0}}{3} U_{Z}^{2}+\delta\left(-\frac{1}{24} U_{Z}^{2}+\left(\frac{1}{3}-\frac{1}{9} U\right) U_{Z Z}-\frac{16}{9} U_{Z Z} G-\frac{2}{3} U_{Z} G_{Z}+\frac{1}{\nu_{0}}\left(\frac{2}{9} U^{2}\right.\right. \\
& \left.-\frac{38}{81} U^{3}+\left(\frac{1}{9} U-\frac{19}{54} U^{2}\right) G+\left(\frac{1}{72}-\frac{17}{108} U\right) G^{2}-\frac{2}{81} G^{3}\right)=0 .
\end{aligned}
$$

From (4.3a) and (4.3b), we find that the coefficients of these two equations do not depend upon the material constant $\mu_{0}$ and only depend upon the geometrical parameters $\delta$ and $\nu_{0}$.

In the following sections, we study the bifurcations of the governing system of ODEs (4.3a) and (4.3b) subjected to some proper end boundary conditions.

\section{Linear bifurcation analysis with sliding boundary conditions}

In this section, we will conduct a linear bifurcation analysis for the coupled ODE system (4.3a) and (4.3b) with sliding boundary conditions. Without loss of generality, we suppose the length of the tube $l$ to be 1 in the following discussions.

We assume sliding boundary conditions at the two ends of the tube, which means the shear stresses on the two end surfaces of the tube are zeros, that is,

$$
S_{Z r}=0 \quad \text { at } \quad Z=0,1 .
$$

We also assume that the two end surfaces of the tube keep flat during the deformation, which leads to

$$
W_{R}(R, Z)=0 \quad \text { at } Z=0,1,
$$

where $W$ is the axial displacement defined in (2.1). Based on the above two equations and adopting the manipulations introduced in Section 3, we obtain the following reduced boundary conditions:

$$
U_{Z}=0, \quad G_{Z}=0 \quad \text { at } \quad Z=0,1 .
$$


We first consider the homogeneous deformation of the elastic tube, that is,

$$
U=U_{h}, \quad G=G_{h} \text { for } \quad 0 \leq Z \leq 1,
$$

where $U_{h}$ and $G_{h}$ are two constants. It is clear that (5.4) satisfy the end boundary conditions (5.3). To determine $U_{h}$ and $G_{h}$, we substitute (5.4) into (4.3a) and (4.3b) to arrive at two algebraic equations:

$$
\begin{aligned}
& \frac{4}{3} U_{h}-\frac{26}{9} U_{h}^{2}+\frac{398}{81} U_{h}^{3}+\left(\frac{1}{3}-\frac{1}{9} U_{h}+\frac{2}{27} U_{h}^{2}\right) G_{h}+\left(-\frac{1}{18}+\frac{1}{54} U_{h}\right) G_{h}^{2}+\frac{2}{81} G_{h}^{3} \\
&+ \rho\left(-\frac{4}{3} U_{h}+\frac{34}{9} U_{h}^{2}-\frac{218}{27} U_{h}^{3}+\left(-\frac{1}{3}+\frac{5}{9} U_{h}-\frac{4}{3} U_{h}^{2}\right) G_{h}+\left(\frac{1}{9}-\frac{1}{3} U_{h}\right) G_{h}^{2}\right. \\
&\left.-\frac{2}{27} G_{h}^{3}\right)=0 \\
& \frac{5}{4} \gamma-\frac{1}{3} U_{h}+\frac{1}{18} U_{h}^{2}-\frac{2}{81} U_{h}^{3}+\left(-\frac{4}{3}+\frac{1}{9} U_{h}-\frac{1}{54} U_{h}^{2}\right) G_{h}+\left(\frac{26}{9}-\frac{2}{27} U_{h}\right) G_{h}^{2}-\frac{398}{81} G_{h}^{3} \\
&+\rho\left(\frac{2}{9} U_{h}^{2}-\frac{38}{81} U_{h}^{3}+\left(\frac{1}{9} U_{h}-\frac{19}{54} U_{h}^{2}\right) G_{h}+\left(\frac{1}{72}-\frac{17}{108} U_{h}\right) G_{h}^{2}-\frac{2}{81} G_{h}^{3}\right)=0,
\end{aligned}
$$

where $\rho=\delta / \nu_{0}$. Note that (5.5a) and (5.5b) are both third-order polynomial equations. Thus obtaining the explicit expressions of $U_{h}$ and $G_{h}$ from these two equations may be very complicated. Obviously, $U_{h}$ and $G_{h}$ should be functions of the engineering stress $\gamma$. As $\gamma$ is a parameter of $O(\varepsilon)$ (see (3.2) and (5.5b)), we consider the asymptotic expressions of $U_{h}$ and $G_{h}$ in terms of $\gamma$. By using the regular perturbation method, we can obtain the following asymptotic expressions for $U_{h}$ and $G_{h}$ :

$$
U_{h}=-\frac{1}{4} \gamma-\frac{13}{32} \gamma^{2}-\frac{125}{128} \gamma^{3}, \quad G_{h}=\gamma+\frac{9}{4} \gamma^{2}+\frac{25}{4} \gamma^{3}
$$

We note that the asymptotic solutions do not depend on $\rho$ (as should be the case for a homogeneous deformation induced by uniaxial compression; thus this can also be regarded as a consistency check for our approach).

In Figure 2, we show the comparisons of the solutions of (5.5a) and (5.5b) with the homogeneous solutions of the original exact equations (2.6a), (2.6b) and (2.7). It can be seen that when $-0.2<\gamma$ $<0.1$, they agree with each other very well. Also, through the comparisons of the asymptotic solutions (5.6) with the solutions of (5.5a) and (5.5b), we find that when $|\gamma|<0.16$, the asymptotic solutions (5.6) are good approximations of the solutions of (5.5a) and (5.5b).

In addition to the constant solutions, there may also exist nontrivial solutions satisfying (4.3a) and (4.3b) and the boundary conditions (5.3) when the external force exceeds a critical value. In the following, we will determine the critical stress values at which there exist nontrivial solutions. First, we set

$$
U=U_{h}+\Delta U, \quad G=G_{h}+\Delta G
$$
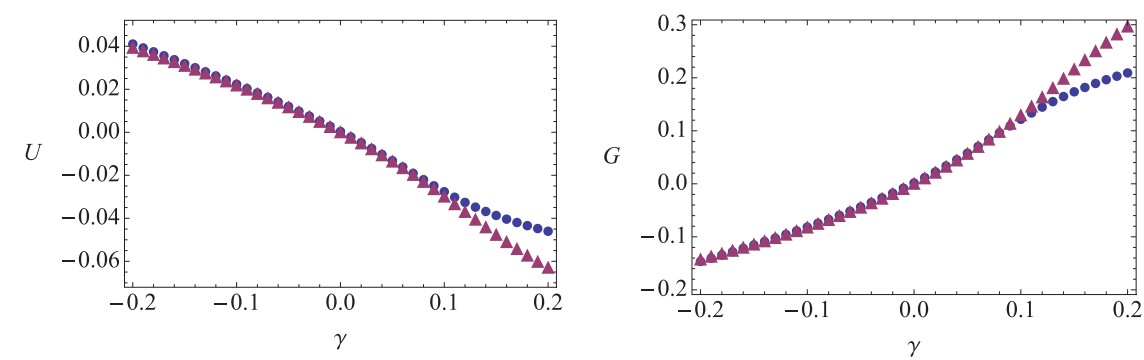

Figure 2. Comparisons of the solutions (circles) of (5.5a) and (5.5b) with the homogeneous solutions (triangles) of (2.6a) to (2.7). 
where $\Delta U$ and $\Delta G$ are two small variations. Substituting (5.7) into (4.3a) and (4.3b) and further dropping the nonlinear terms, we have

$$
\begin{gathered}
c_{1} \Delta G_{Z Z}+c_{2} \Delta G+c_{3} \Delta U_{Z Z}+c_{4} \Delta U=0, \\
c_{1} \Delta U_{Z Z}+c_{5} \Delta U+c_{6} \Delta G=0,
\end{gathered}
$$

where $c_{i}(i=1,2, \ldots, 26)$ in the above equations and the following equations are constants dependent on $U_{h}, G_{h}, \rho$ and $\nu_{0}$.

From (5.3), $\Delta U$ and $\Delta G$ should satisfy the following boundary conditions:

$$
\Delta U_{Z}=0, \quad \Delta G_{Z}=0 \quad \text { at } \quad Z=0,1 .
$$

To find the critical stress values at which there exist nontrivial solutions of $U$ and $G$ satisfying (4.3a), (4.3b) and (5.3) is to find the critical stress values at which there exist nontrivial solutions of $\Delta U$ and $\Delta G$ for the linear system (5.8a) and (5.8b) with the boundary conditions (5.9). We suppose that $\Delta U$ and $\Delta G$ have the following general forms:

$$
\Delta U(Z)=A e^{\lambda Z}, \quad \Delta G(Z)=B e^{\lambda Z},
$$

where $A, B$ and $\lambda$ are constants. Substituting (5.10) into (5.8a) and (5.8b), we have the following linear system:

$$
M_{1}\left(\begin{array}{l}
A \\
B
\end{array}\right)=\left(\begin{array}{ll}
m_{11} & m_{12} \\
m_{21} & m_{22}
\end{array}\right)\left(\begin{array}{l}
A \\
B
\end{array}\right)=0
$$

where $m_{i j}(i, j=1,2)$ are in terms of $\lambda, U_{h}, G_{h}, \rho$ and $\nu_{0}$ which are not shown for brevity.

To find nontrivial solutions of (5.8a) and (5.8b), we need Det $M_{1}=0$, which yields the characteristic equation of the ODE system of (5.8a) and (5.8b),

$$
\psi_{3} \lambda^{4}+\psi_{2} \lambda^{2}+\psi_{1}=0
$$

where $\psi_{i}(i=1,2,3)$ are constants dependent on $c_{i}(i=1, \ldots, 6)$. Equation (5.12) is a fourth-order polynomial equation, thus there are four roots. As (5.12) only depends on the even orders of $\lambda$, the four roots can be divided into two pairs. The two roots in each pair are negatives of each other. We can classify the four roots of equation (5.12) into the following three different cases: (1) there are four real roots; (2) there are four purely imaginary roots; (3) there are four single complex roots (both the real parts and the imaginary parts of these complex roots are non-zero). In cases (1) and (2), there may exist twofold repeated roots at some special points.

Substituting (5.6) into (5.12), we find that the coefficients of (5.12) depend only on the stress value $\gamma$ and two parameters $\rho$ and $\nu_{0}$. In derivations of (3.6a) and (3.6b), some restrictions on the geometrical parameters have been imposed (see Figure 1). On the other hand, to ensure the validity of the asymptotic expressions of homogeneous solutions (5.6), we require $|\gamma|<0.16$. Under these constraints and through some calculations, we find that there are two particular stress values $\gamma_{m}(<0)$ and $\gamma_{p}(>0)$ (dependent on $\rho$ ). According to these two values, as $\gamma$ varies, types of roots of (5.12) can be divided into five cases, listed in Table 1.

Table I. Types of roots of (5.12) varying with $\gamma$.
(i) $-0.16<\gamma<\gamma_{m}$
(ii) $\gamma=\gamma_{m}$
(iii) $\gamma_{m}<\gamma<\gamma_{p}$
(iv) $\gamma=\gamma_{p}$
(v) $\gamma_{p}<\gamma<0.16$

Two different pairs of purely imaginary conjugate roots Two pairs of repeated purely imaginary roots Four single complex roots Two pairs of repeated real roots Four single real roots 
In this paper, we study the compression problem (which implies $\gamma<0$ ). Then both cases (i) and (ii) are possible. We first study case (ii) and we will show that this case will not happen in general. In this case $\gamma=\gamma_{m}$, we let the two pairs of repeated roots be $\pm \lambda_{1}$ i and the solution expressions for (5.8a) and (5.8b) are given by

$$
\left\{\begin{array}{l}
\Delta U=\left(A_{1}+A_{3} Z\right) \cos \left(\lambda_{1} Z\right)+\left(A_{2}+A_{4} Z\right) \sin \left(\lambda_{1} Z\right), \\
\Delta G=\left(B_{1}+B_{3} Z\right) \cos \left(\lambda_{1} Z\right)+\left(B_{2}+B_{4} Z\right) \sin \left(\lambda_{1} Z\right)
\end{array}\right.
$$

where $A_{i}$ and $B_{i}(i=1,2,3,4)$ are real constants.

By substituting (5.13) into (5.8a) and (5.8b) and comparing the coefficients of $\cos (\lambda Z), \sin (\lambda Z)$, $Z \cos (\lambda Z)$ and $Z \sin (\lambda Z)$, we have

$$
\begin{aligned}
& A_{1}=b_{2} B_{4}, \quad A_{2}=-b_{2} B_{3}, \quad A_{3}=b_{1} B_{3}, \quad A_{4}=b_{1} B_{4}, \\
& B_{1}=b_{3} B 4, \quad B_{2}=-b_{3} B_{3},
\end{aligned}
$$

where $b_{i}(i=1,2,3)$ are in terms of $U_{h}, G_{h}, \rho, \nu_{0}$ and $\lambda_{1}$ whose expressions are omitted for brevity. If we further substitute (5.13) and (5.14) into the boundary condition (5.9), we get a linear system for $B_{3}$ and $B_{4}$ and it is easy to find that there are only trivial solutions $B_{3}=B_{4}=0$ except when $b_{2}=b_{1} b_{3}$. However, this only occurs in a very special circumstance (one needs $\gamma=\gamma_{m}$ but also $b_{2}=b_{1} b_{3}$ ).

In this paper, we shall concentrate on case (i) and in this case we denote the four purely imaginary roots of (5.12) as

$$
\pm \lambda_{1} \mathrm{i}= \pm\left(\frac{\psi_{2}+\sqrt{\psi_{2}^{2}-4 \psi_{1} \psi_{3}}}{2 \psi_{3}}\right)^{\frac{1}{2}} \mathrm{i}, \quad \pm \lambda_{2} \mathrm{i}= \pm\left(\frac{\psi_{2}-\sqrt{\psi_{2}^{2}-4 \psi_{1} \psi_{3}}}{2 \psi_{3}}\right)^{\frac{1}{2}} \mathrm{i}
$$

Without loss of generality, we take both $\lambda_{1}$ and $\lambda_{2}$ to be positive. For this case, the solution expressions for $\Delta U$ and $\Delta G$ can be written as

$$
\left\{\begin{array}{l}
\Delta U=A_{1} \cos \left(\lambda_{1} Z\right)+A_{2} \sin \left(\lambda_{1} Z\right)+A_{3} \cos \left(\lambda_{2} Z\right)+A_{4} \sin \left(\lambda_{2} Z\right) \\
\Delta G=B_{1} \cos \left(\lambda_{1} Z\right)+B_{2} \sin \left(\lambda_{1} Z\right)+B_{3} \cos \left(\lambda_{2} Z\right)+B_{4} \sin \left(\lambda_{2} Z\right)
\end{array}\right.
$$

where $A_{i}$ and $B_{i}(i=1,2,3,4)$ are real constants.

By substituting (5.16) into (5.8a) and (5.8b) and comparing the coefficients of $\cos \left(\lambda_{i} Z\right)$ and $\sin \left(\lambda_{i} Z\right)$ $(i=1,2)$, we have

$$
B_{1}=f\left(\lambda_{1}\right) A_{1}, \quad B_{2}=f\left(\lambda_{1}\right) A_{2}, \quad B_{3}=f\left(\lambda_{2}\right) A_{3}, \quad B_{4}=f\left(\lambda_{2}\right) A_{4},
$$

where $f(\lambda)=\left(c_{1} \lambda^{2}-c_{5}\right) / c_{6}$.

Substituting (5.16) into the boundary condition (5.9), and from (5.17), we have

$$
M_{2}\left(\begin{array}{llll}
A_{1} & A_{2} & A_{3} & A_{4}
\end{array}\right)^{\mathrm{T}}=0,
$$

where

$$
M_{2}=\left(\begin{array}{cccc}
0 & \lambda_{1} & 0 & \lambda_{2} \\
-\lambda_{1} \sin \lambda_{1} & \lambda_{1} \cos \lambda_{1} & -\lambda_{2} \sin \lambda_{2} & \lambda_{2} \cos \lambda_{2} \\
0 & f\left(\lambda_{1}\right) \lambda_{1} & 0 & f\left(\lambda_{2}\right) \lambda_{2} \\
-f\left(\lambda_{1}\right) \lambda_{1} \sin \lambda_{1} & f\left(\lambda_{1}\right) \lambda_{1} \cos \lambda_{1} & -f\left(\lambda_{2}\right) \lambda_{2} \sin \lambda_{2} & f\left(\lambda_{2}\right) \lambda_{2} \cos \lambda_{2}
\end{array}\right) .
$$

To find nontrivial solutions of $\Delta U$ and $\Delta G$, we need

$$
\operatorname{Det} M_{2}=\left(f\left(\lambda_{1}\right)-f\left(\lambda_{2}\right)\right)^{2} \lambda_{1}^{2} \lambda_{2}^{2} \sin \lambda_{1} \sin \lambda_{2}=0 .
$$


It is easy to see that (5.19) holds in two cases: (i) $f\left(\lambda_{1}\right)=f\left(\lambda_{2}\right)$; (ii) $\lambda_{1}=n \pi$ or $\lambda_{2}=n \pi$ (where $n$ is a positive integer).

We have tested some parameter values and find that in general $f\left(\lambda_{1}\right)=f\left(\lambda_{2}\right)$ together with (5.12) cannot be satisfied. Therefore, in the following we consider the bifurcation points corresponding to $\lambda=$ $n \pi$ i. In the case of $\lambda_{1}=n \pi$ or $\lambda_{2}=n \pi$, from (5.18), it can be deduced that the solution (5.16) has the following reduced form:

$$
\Delta U=C_{1} \cos (n \pi Z), \quad \Delta G=f(n \pi) C_{1} \cos (n \pi Z),
$$

where $C_{1}$ is a constant.

Substituting $\lambda=n \pi \mathrm{i}$ into (5.12), we have

$$
\psi_{3} \pi^{4} n^{4}-\psi_{2} \pi^{2} n^{2}+\psi_{1}=0
$$

which provides the relationship between the critical stress value $\gamma_{c}$, the mode number $n$ and the geometrical parameters $\rho$ and $\nu_{0}$. Actually, substituting the asymptotic expressions of $U_{h}$ and $G_{h}$ (see (5.6)) into the above equation, we have

$$
g_{3} \pi^{4} n^{4}-g_{2} \pi^{2} n^{2}+g_{1}=0
$$

where

$$
\left\{\begin{aligned}
g_{1}= & \frac{135}{\left(\rho \nu_{0}\right)^{2}}-\frac{135}{\rho \nu_{0}^{2}}+\left(-\frac{945}{2\left(\rho \nu_{0}\right)^{2}}+\frac{945}{2 \rho \nu_{0}^{2}}\right) \gamma_{c}+\left(-\frac{405}{4\left(\rho \nu_{0}\right)^{2}}+\frac{405}{4 \rho \nu_{0}^{2}}\right) \gamma_{c}^{2}+\left(\frac{46,665}{16\left(\rho \nu_{0}\right)^{2}}-\frac{75}{32 \nu_{0}^{2}}-\frac{46,365}{16 \rho \nu_{0}^{2}}\right) \gamma_{c}^{3}, \\
g_{2}= & -\frac{9}{\nu_{0}}+\left(\frac{135}{2 \rho \nu_{0}}+\frac{99}{2 \nu_{0}}-\frac{135}{\rho^{2} \nu_{0}}\right) \gamma_{c}+\left(-\frac{4545}{32 \rho \nu_{0}}+\frac{789}{8 \nu_{0}}+\frac{4365}{16 \rho^{2} \nu_{0}}\right) \gamma_{c}^{2} \\
& +\left(-\frac{36,585}{256 \rho \nu_{0}}+\frac{1023}{4 \nu_{0}}+\frac{37,395}{128 \rho^{2} \nu_{0}}\right) \gamma_{c}^{3}, \\
g_{3}= & 9-\frac{189}{2} \gamma_{c}+\frac{69}{2} \gamma_{c}^{2}+\frac{8433}{16} \gamma_{c}^{3} .
\end{aligned}\right.
$$

Equation (5.22) explicitly provides the relation between the critical stress value $\gamma_{c}$, the mode number $n$ and the geometric parameters $\rho$ and $\nu_{0}$. For given mode number $n$ and $\nu_{0}$, it determines the relation between $\gamma_{c}$ and $\rho$. For example, for $\nu_{0}=0.1$ and 0.22 , we show the dependence of these two quantities for some different mode numbers $n$ in Figure 3. We find that once mode number $n$ is known for a given $\nu_{0}$, the critical stress $\gamma_{c}$ is always a decreasing function of $\rho$ as can be seen from Figure 3.

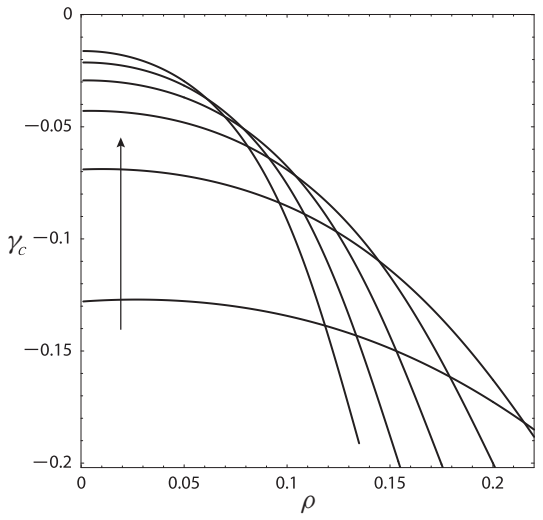

(a) $\nu_{0}=0.1$

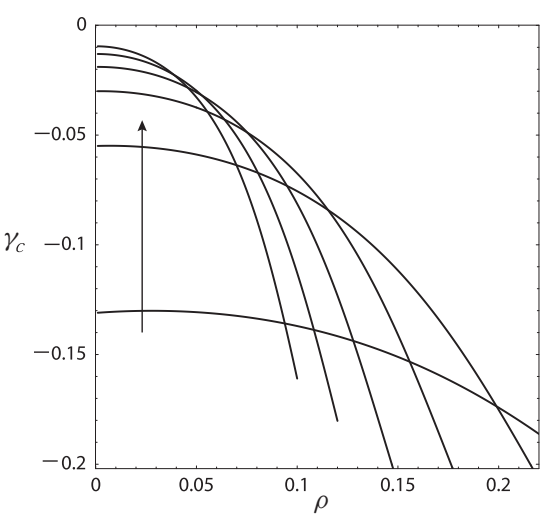

(b) $\nu_{0}=0.22$

Figure 3. The plots of $\gamma_{c}$ vs $\rho$ (a measure of the tube thickness) for mode numbers $(a) 3 \leq n \leq 8$ and (b) $2 \leq n \leq 7$. The arrow indicates the direction of increasing of $n$. 


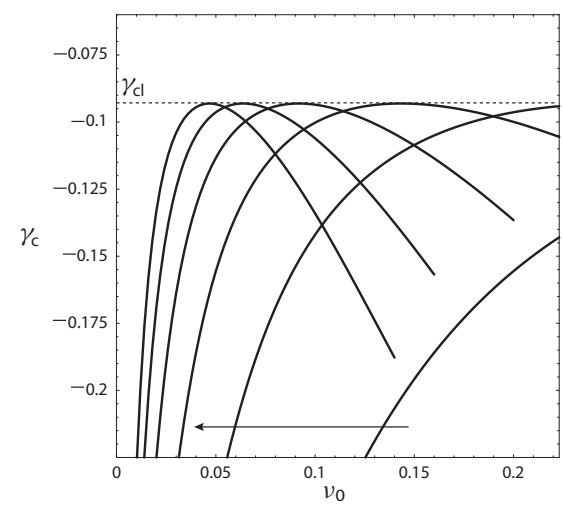

(a) $\rho=0.13$

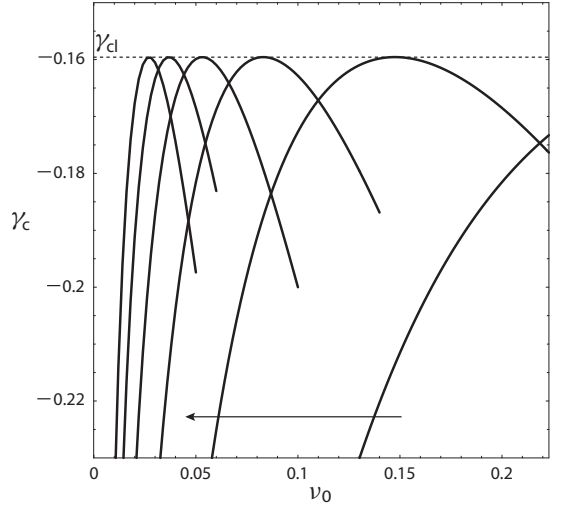

(b) $\rho=0.2$

Figure 4. The plots of $\gamma_{c}$ vs $\nu_{0}$ (a measure of the tube slenderness) for mode numbers $2 \leq n \leq 7$. The arrow indicates the direction of increasing of $n$.

Also, from Figure 3, we see that two curves can intersect at a particular value of $\rho$, in other words, two bifurcation mode numbers can correspond to the same critical stress value. However, it is not found that more than two modes can correspond to the same critical stress value. Denote the intersection point of the $n$ th-mode and $k$ th-mode curves as $\left(\rho^{(n, k)}, \gamma_{c}^{(n, k)}\right)$; we can observe the following pattern in Figure 3.

For a geometrical parameter $\rho$, suppose that the $(k+1)$ th mode has the smallest critical stress value $\left|\gamma_{c}^{s}\right|$ (i.e. the first mode number is $\left.k+1\right)$. If for a particular $\rho$ it happens that $\left(\gamma_{c}^{s}, \rho\right)$ is an intersection point, then $\gamma_{c}^{s}$ has to be $\gamma_{c}^{(k, k+1)}$, in other words, it has to be the intersection point of the neighboringmode curves. For example, for $\nu_{0}=0.1$ and $\rho=0.0784001$, the smallest $\left|\gamma_{c}^{s}\right|$ is attained at the seventhmode curve. It happens that $\left(\gamma_{c}^{s}, \rho\right)$ is an intersection point for this particular $\rho$, and we can see it is the intersection point of the seventh-mode and sixth-mode curves. When this happens, from (5.18) we can deduce that the solution (5.16) has the following reduced form:

$$
\left\{\begin{array}{l}
\Delta U=C_{1} \cos (k \pi Z)+C_{2} \cos ((k+1) \pi Z), \\
\Delta G=f(n \pi) C_{1} \cos (k \pi Z)+f((k+1) \pi) C_{2} \cos ((k+1) \pi Z),
\end{array}\right.
$$

where $C_{i}(i=1,2)$ are constants.

This observation is very useful, since some interesting phenomena can arise when there are two modes for the same critical stress. These will be addressed in later sections.

In Figure 3, for a given $\nu_{0}$, we can deduce the first mode number from the value of $\rho$. Once $\nu_{0}$ is given, we can calculate all $\rho^{(k, k+1)}(k=1,2,3, \ldots)$. One observation from Figure 3 is that for a given $\rho$ lying between $\rho^{(k, k+1)}$ and $\rho^{(k+1, k+2)},\left|\gamma_{c}^{s}\right|$ is always attained at the $(k+1)$ th-mode curve. So, the first bifurcation mode is determined. We also observe that the difference between $\rho^{(k, k+1)}$ and $\rho^{(k+1, k+2)}$ becomes smaller as $\rho$ decreases. Thus, for a thin tube it easily happens that two bifurcation modes correspond to the same critical stress.

Similarly, when the mode number $n$ and $\rho$ are given, we can determine the relation between $\gamma_{c}$ and $\nu_{0}$ from (5.22). This relation is shown in Figure 4 for some different mode numbers $n$. This figure can be used to find the first mode number from the value of $\nu_{0}$. Denote the intersection point of the $n$ th-mode and $k$ th-mode curves as $\left(\nu_{0}^{(n, k)}, \gamma_{c}^{(n, k)}\right)$ and suppose the $(k+1)$ th mode has the smallest critical stress value $\left|\gamma_{c}^{s}\right|$. We can observe the following pattern in Figure 4. For a given $\nu_{0}$ lying between $\nu_{0}^{(k, k+1)}$ and $\nu_{0}^{(k+1, k+2)},\left|\gamma_{c}^{s}\right|$ is attained at the $(k+1)$ th-mode curve. We also observe that the smallest values of $\left|\gamma_{c}^{s}\right|$ for each mode number are almost the same (see the horizontal dashed lines in Figure 4). This implies that for a given $\rho$ the critical stress has a uniform lower bound $\gamma_{c l}$ for all $\nu_{0}$; see Figure 4 . In particular, if both the inner and outer radii of the tube are fixed (then $\rho$ is fixed), the critical stress has a uniform lower bound for all different tube lengths. This implies that, when the compression stress is below $\gamma_{c l}$, no bifurcation to axisymmetrical modes can take place no matter what the length of the tube is. 


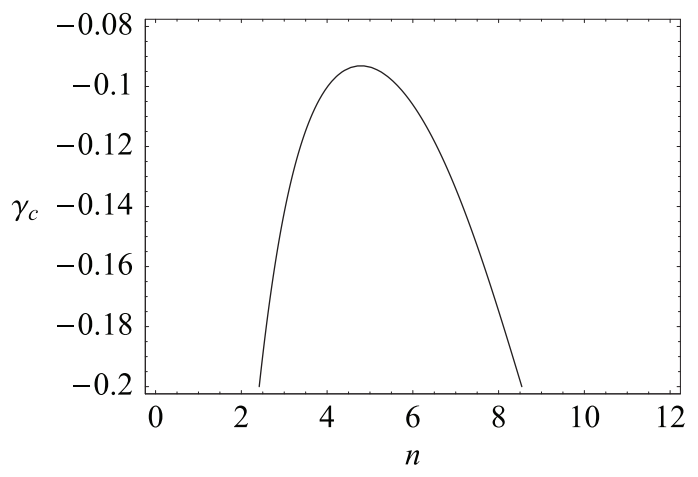

Figure 5. The curve of the critical stress $\gamma_{c}$ as a continuous function of $n$ when $\nu_{0}=0.1$ and $\rho=0.13$.

Of course, all the conclusions are based on the constraints of the geometrical parameters imposed at the end of Section 3.

When both $\nu_{0}$ and $\rho$ are given, one can find the first mode number from the curve of the critical stress by regarding it as a continuous function of the mode number, which is shown in Figure 5 for $\nu_{0}=0.1$ and $\rho=0.13$. From this figure, we find that the first mode number should be $n=5$, which can also be observed from Figure 3(a) or Figure 4(a).

In the above linear analysis, we have presented some relations between the critical stress value, mode number and geometrical parameters. In the following section, we will obtain the approximate postbifurcation solutions by using the method of multiple scales.

\section{Analytical post-bifurcation solutions and discussions}

A linear bifurcation analysis can yield the critical loads for bifurcation and the eigenmodes. However, one has to conduct a nonlinear analysis to understand the post-bifurcation behavior. However, it seems impossible to find the exact analytical solutions for the complicated coupled nonlinear ODE system (4.3a), (4.3b) and (5.3). When the external stress is close to the critical stress value $\gamma_{c}$, as the buckling amplitude is small, it is possible to find the approximate analytical solutions by perturbation methods. In this section, some nonlinear bifurcation analyses will be carried out in the vicinity of the bifurcation points. In other words, we consider the approximate analytical solutions for the post-bifurcation states governed by the coupled system (4.3a) and (4.3b) with the boundary conditions (5.3) at near-critical loads.

To proceed with the perturbation method, we first need to introduce a small parameter. Let $U_{h}$ and $G_{h}$ denote the solutions for a homogeneous deformation of (4.3a) and (4.3b) when $\gamma=\gamma_{c}$; in other words, $U_{h}$ and $G_{h}$ satisfy (5.5a) and (5.5b) with $\gamma=\gamma_{c}$. For the present problem, we seek the solutions in the form of the following perturbation expansions:

$$
\begin{aligned}
& U=U_{h}+\zeta U^{(0)}+\zeta^{2} U^{(1)}+\zeta^{3} U^{(2)}+\ldots, \\
& G=G_{h}+\zeta G^{(0)}+\zeta^{2} G^{(1)}+\zeta^{3} G^{(2)}+\ldots \\
& \gamma=\gamma_{c}+\Delta \gamma, \quad \Delta \gamma=\zeta^{2} \hat{\gamma}
\end{aligned}
$$

where $\zeta$ is a small positive parameter representing the amplitude of the difference $U-U_{h}$ and $\Delta \gamma=\zeta^{2} \hat{\gamma}$ is the incremental stress value on $\gamma_{c}$. The above assumptions imply that the amplitude of $U-U_{h}$ is of the same order as $G-G_{h}$ and is of order $\sqrt{|\Delta \gamma|}$. This order relation is determined by requiring that $\hat{\gamma}$ should appear in the amplitude equation for $U^{(0)}$, which will become clear in the following derivations.

In the following, we use the method of multiple scales with two variables (see [31]) to find the approximate analytical solutions of (4.3a) and (4.3b). We first introduce

$$
Z_{0}=Z, \quad Z_{1}=\zeta^{2} Z
$$


Substituting (6.1) into (4.3a) and (4.3b) and equating the coefficients of the like powers of $\zeta$ to zero, we obtain a system of equations. As expected, the coefficients of $\zeta^{0}$ yield (5.5a) and (5.5b) with $\gamma=\gamma_{c}$, which are automatically satisfied.

(1) From the coefficients of $\zeta$, we have

$$
\begin{gathered}
c_{1} G_{Z_{0} Z_{0}}^{(0)}+c_{2} G^{(0)}+c_{3} U_{Z_{0} Z_{0}}^{(0)}+c_{4} U^{(0)}=0, \\
c_{1} U_{Z_{0} Z_{0}}^{(0)}+c_{5} U^{(0)}+c_{6} G^{(0)}=0 .
\end{gathered}
$$

Note that the above equations are similar to (5.8a) and (5.8b).

One can solve $G^{(0)}$ from the second equation, which is then substituted into the first equation to give

$$
\psi_{3} U_{Z_{0} Z_{0} Z_{0} Z_{0}}^{(0)}+\psi_{2} U_{Z_{0} Z_{0}}^{(0)}+\psi_{1} U^{(0)}=0
$$

The characteristic equation of (6.4) is simply (5.12), as expected. Therefore, the roots are given by (5.15). Then the solution expressions of $U^{(0)}$ and $G^{(0)}$ are

$$
\begin{gathered}
U^{(0)}=K_{1}\left(Z_{1}\right) e^{\mathrm{i} \lambda_{1} Z_{0}}+K_{2}\left(Z_{1}\right) e^{\mathrm{i} \lambda_{2} Z_{0}}+C C, \\
G^{(0)}=f\left(\lambda_{1}\right) K_{1}\left(Z_{1}\right) e^{\mathrm{i} \lambda_{1} Z_{0}}+f\left(\lambda_{2}\right) K_{2}\left(Z_{1}\right) e^{\mathrm{i} \lambda_{2} Z_{0}}+C C,
\end{gathered}
$$

where $C C$ denotes the complex conjugate of the previous terms, and $K_{1}\left(Z_{1}\right)$ and $K_{2}\left(Z_{1}\right)$ are two functions to be determined.

(2) From the coefficients of $\zeta^{2}$, we have

$$
\begin{aligned}
& c_{1} G_{Z_{0} Z_{0}}^{(1)}+c_{2} G^{(1)}+c_{3} U_{Z_{0} Z_{0}}^{(1)}+c_{4} U^{(1)}-\frac{16}{9} \rho \nu_{0}\left(G_{Z_{0}}^{(0)}\right)^{2}-\frac{16}{9} \rho \nu_{0} G^{(0)} G_{Z_{0} Z_{0}}^{(0)}-\frac{1}{9} \rho \nu_{0} G_{Z_{0} Z_{0}}^{(0)} U^{(0)} \\
& \quad-\frac{19}{36} \rho \nu_{0}\left(U_{Z_{0}}^{(0)}\right)^{2}+c_{7}\left(U^{(0)}\right)^{2}+c_{8} G^{(0)} U^{(0)}+c_{9}\left(G^{(0)}\right)^{2}+c_{10} G_{Z_{0}}^{(0)} U_{Z_{0}}^{(0)}+c_{11} G^{(0)} U_{Z_{0} Z_{0}}^{(0)} \\
& \quad+c_{12} U^{(0)} U_{Z_{0} Z_{0}}^{(0)}-\frac{1}{6} \nu_{0}\left(U_{Z_{0}}^{(0)}\right)^{2}=0, \\
& c_{1} U_{Z_{0} Z_{0}}^{(1)}+c_{5} U^{(1)}+c_{6} G^{(1)}-\frac{2}{3} \rho \nu_{0} G_{Z_{0}}^{(0)} U_{Z_{0}}^{(0)}-\frac{1}{24} \rho \nu_{0}\left(U_{Z_{0}}^{(0)}\right)^{2}-\frac{16}{9} \rho \nu_{0} G^{(0)} U_{Z_{0} Z_{0}}^{(0)}-\frac{2}{3} \nu_{0}\left(U_{Z_{0}}^{(0)}\right)^{2} \\
& -\frac{1}{9} \rho \nu_{0} U^{(0)} U_{Z_{0} Z_{0}}^{(0)}+c_{13}\left(U^{(0)}\right)^{2}+c_{14} G^{(0)} U^{(0)}+c_{15}\left(G^{(0)}\right)^{2}=-\frac{5}{4} \hat{\gamma} .
\end{aligned}
$$

From the second equation, $G^{(1)}$ can be expressed in terms of $U^{(1)}$. We substitute the expression into the first equation to yield an equation for $U^{(1)}$ which gives

$$
\begin{aligned}
U^{(1)}= & M_{1}\left(Z_{1}\right) e^{\mathrm{i} \lambda_{1} Z_{0}}+M_{2}\left(Z_{1}\right) e^{\mathrm{i} \lambda_{2} Z_{0}}+\alpha_{1} K_{1}^{2}\left(Z_{1}\right) e^{2 \mathrm{ii} \lambda_{1} Z_{0}}+\alpha_{2} K_{2}^{2}\left(Z_{1}\right) e^{2 \mathrm{i} \lambda_{2} Z_{0}} \\
& +\alpha_{3} K_{1}\left(Z_{1}\right) K_{2}\left(Z_{1}\right) e^{\mathrm{i}\left(\lambda_{1}+\lambda_{2}\right) Z_{0}}+\alpha_{4} K_{1}^{*}\left(Z_{1}\right) K_{2}\left(Z_{1}\right) e^{\mathrm{i}\left(-\lambda_{1}+\lambda_{2}\right) Z_{0}} \\
& +C C+\alpha_{0} \hat{\gamma}+\alpha_{5} K_{1}\left(Z_{1}\right) K_{1}^{*}\left(Z_{1}\right)+\alpha_{6} K_{2}\left(Z_{1}\right) K_{2}^{*}\left(Z_{1}\right)
\end{aligned}
$$

where $M_{1}\left(Z_{1}\right)$ and $M_{2}\left(Z_{1}\right)$ are two unknown functions, and $\alpha_{i}(i=0,1, \ldots, 6)$ are constants related to $U_{h}, G_{h}, \rho$ and $\nu_{0}$. Also, we can obtain the solution expression of $G^{(1)}$ :

$$
\begin{aligned}
G^{(1)}= & f\left(\lambda_{1}\right) M_{1}\left(Z_{1}\right) e^{\mathrm{i} \lambda_{1} Z_{0}}+f\left(\lambda_{2}\right) M_{2}\left(Z_{1}\right) e^{\mathrm{i} \lambda_{2} Z_{0}}+\beta_{1} K_{1}^{2}\left(Z_{1}\right) e^{2 \mathrm{i} \lambda_{1} Z_{0}}+\beta_{2} K_{2}^{2}\left(Z_{1}\right) e^{2 \mathrm{i} \lambda_{2} Z_{0}} \\
& +\beta_{3} K_{1}\left(Z_{1}\right) K_{2}\left(Z_{1}\right) e^{\mathrm{i}\left(\lambda_{1}+\lambda_{2}\right) Z_{0}}+\beta_{4} K_{1}\left(Z_{1}\right) K_{2}^{*}\left(Z_{1}\right) e^{\mathrm{i}\left(\lambda_{1}-\lambda_{2}\right) Z_{0}}+C C \\
& +\beta_{0} \hat{\gamma}+\beta_{5} K_{1}\left(Z_{1}\right) K_{1}^{*}\left(Z_{1}\right)+\beta_{6} K_{2}\left(Z_{1}\right) K_{2}^{*}\left(Z_{1}\right),
\end{aligned}
$$

where $\beta_{i}(i=0,1, \ldots, 6)$ are constants related to $U_{h}, G_{h}, \rho$ and $\nu_{0}$.

(3) From the coefficients of $\zeta^{3}$, we have 


$$
\begin{aligned}
& c_{1} G_{Z_{0} Z_{0}}^{(2)}+c_{2} G^{(2)}+c_{3} U_{Z_{0} Z_{0}}^{(2)}+c_{4} U^{(2)}-\frac{32}{9} \rho \nu_{0} G_{Z_{0}}^{(0)} G_{Z_{0}}^{(1)}+2 c_{1} G_{Z_{0} Z_{1}}^{(0)}-\frac{16}{9} \rho \nu_{0} G_{Z_{0} Z_{0}}^{(1)} G^{(0)} \\
& \quad-\frac{1}{9} \rho \nu_{0} G_{Z_{0} Z_{0}}^{(0)}\left(16 G^{(1)}+U^{(1)}\right)+U_{Z_{0} Z_{0}}^{(1)}\left(c_{11} G^{(0)}+c_{12} U^{(0)}\right)+c_{21}\left(U^{(0)}\right)^{3}+c_{22} G^{(0)}\left(U^{(0)}\right)^{2} \\
& +c_{23}\left(G^{(0)}\right)^{2} U^{(0)}+c_{24}\left(G^{(0)}\right)^{3}+2 c_{7} U^{(0)} U^{(1)}+c_{8} G^{(1)} U^{(0)}+c_{8} G^{(0)} U^{(1)}+2 c_{9} G^{(0)} G^{(1)} \\
& +c_{10} G_{Z_{0}}^{(1)} U_{Z_{0}}^{(0)}+c_{10} G_{Z_{1}}^{(0)} U_{Z_{0}}^{(0)}+c_{10} G_{Z_{0}}^{(0)} U_{Z_{0}}^{(1)}+c_{10} G_{Z_{0}}^{(0)} U_{Z_{0}}^{(1)}+c_{25} U_{Z_{0}}^{(0)} U_{Z_{0}}^{(1)}+2 c_{3} U_{Z_{0} Z_{1}}^{(0)} \\
& +U_{Z_{0} Z_{0}}^{(0)}\left(\rho \nu_{0}\left(\frac{7}{12} G^{(1)}-\frac{1}{6} U^{(1)}\right)-\frac{1}{3} \nu_{0}\left(4 G^{(1)}+U^{(1)}\right)\right)-\frac{1}{9} \rho \nu_{0} G_{Z_{0} Z_{0}}^{(1)} U^{(0)}=0, \\
& c_{1} U_{Z_{0} Z_{0}}^{(2)}+c_{5} U^{(2)}+c_{6} G^{(2)}-\frac{2}{3} \rho \nu_{0} G_{Z_{0}}^{(1)} U_{Z_{0}}^{(0)}-\frac{1}{9} \rho \nu_{0}\left(16 G^{(1)}+U^{(1)}\right) U_{Z_{0} Z_{0}}^{(0)}-\frac{2}{3} \rho \nu_{0} G_{Z_{0}}^{(0)} U_{Z_{0}}^{(1)} \\
& -\frac{1}{9} \rho \nu_{0}\left(16 G^{(0)}+U^{(0)}\right) U_{Z_{0} Z_{0}}^{(1)}+2 c_{1} U_{Z_{0} Z_{1}}^{(0)}+c_{16}\left(U^{(0)}\right)^{3}+c_{17} G^{(0)}\left(U^{(0)}\right)^{2}+c_{18}\left(G^{(0)}\right)^{2} U^{(0)} \\
& +c_{19}\left(G^{(0)}\right)^{3}+2 c_{13} U^{(0)} U^{(1)}+c_{14} G^{(1)} U^{(0)}+c_{14} G^{(0)} U^{(1)}+2 c_{15} G^{(0)} G^{(1)}+c_{20} U_{Z_{0}}^{(0)} U_{Z_{0}}^{(1)}=0 .
\end{aligned}
$$

We can express $G^{(2)}$ in terms of $U^{(2)}$ from the second equation and then substitute the expression into the first equation to yield an equation for $U^{(2)}$. To remove secular terms, we set $M_{1}\left(Z_{1}\right)=M_{2}\left(Z_{1}\right)=0$ and

$$
\left\{\begin{array}{l}
s_{0} \hat{\gamma} K_{1}\left(Z_{1}\right)+s_{1} K_{1}^{2}\left(Z_{1}\right) K_{1}^{*}\left(Z_{1}\right)+s_{2} K_{1}\left(Z_{1}\right) K_{2}\left(Z_{1}\right) K_{2}^{*}\left(Z_{1}\right)+\mathrm{i} s_{3} \frac{d K_{1}}{d Z_{1}}=0, \\
t_{0} \hat{\gamma} K_{2}\left(Z_{1}\right)+t_{1} K_{2}^{2}\left(Z_{1}\right) K_{2}^{*}\left(Z_{1}\right)+t_{2} K_{2}\left(Z_{1}\right) K_{1}\left(Z_{1}\right) K_{1}^{*}\left(Z_{1}\right)+\mathrm{i} t_{3} \frac{d K_{2}}{d Z_{1}}=0,
\end{array}\right.
$$

where $s_{i}$ and $t_{i}(i=0,1,2,3)$ are constants related to $U_{h}, G_{h}, \rho$ and $\nu_{0}$.

Substituting $K_{1}\left(Z_{1}\right)=R_{1}\left(Z_{1}\right) e^{\mathrm{i} \theta_{1}\left(Z_{1}\right)}$ and $K_{2}\left(Z_{1}\right)=R_{2}\left(Z_{1}\right) e^{\mathrm{i} \theta_{2}\left(Z_{1}\right)}$ into (6.10), we obtain

$$
R_{1}\left(Z_{1}\right)=R_{1} \text { (constant), } \quad R_{2}\left(Z_{1}\right)=R_{2} \text { (constant) }
$$

and

$$
R_{1}\left(s_{0} \hat{\gamma}+s_{1} R_{1}^{2}+s_{2} R_{2}^{2}-s_{3} \frac{d \theta_{1}}{d Z_{1}}\right)=0, \quad R_{2}\left(t_{0} \hat{\gamma}+t_{1} R_{2}^{2}+t_{2} R_{1}^{2}-t_{3} \frac{d \theta_{2}}{d Z_{1}}\right)=0
$$

which provide the equations for the amplitudes of post-bifurcation solutions when $\theta_{1}$ and $\theta_{2}$ are determined. From (6.12), we also have

$$
\begin{array}{ll}
\theta_{1}\left(Z_{1}\right)=\frac{1}{s_{3}}\left(s_{0} \hat{\gamma}+s_{1} R_{1}^{2}+s_{2} R_{2}^{2}\right) Z_{1}+\phi_{1}, & \text { if } \quad R_{1} \neq 0, \\
\theta_{2}\left(Z_{1}\right)=\frac{1}{t_{3}}\left(t_{0} \hat{\gamma}+t_{1} R_{2}^{2}+t_{2} R_{1}^{2}\right) Z_{1}+\phi_{2}, \quad \text { if } \quad R_{2} \neq 0,
\end{array}
$$

where $\phi_{1}$ and $\phi_{2}$ are two integration constants.

To summarize, after the above calculations we have obtained the expressions for the first two terms:

$$
\left\{\begin{array}{l}
U^{(0)}=2 R_{1} \cos \left(\lambda_{1} Z_{0}+\theta_{1}\left(Z_{1}\right)\right)+2 R_{2} \cos \left(\lambda_{2} Z_{0}+\theta_{2}\left(Z_{1}\right)\right) \\
G^{(0)}=2 f\left(\lambda_{1}\right) R_{1} \cos \left(\lambda_{1} Z_{0}+\theta_{1}\left(Z_{1}\right)\right)+2 f\left(\lambda_{2}\right) R_{2} \cos \left(\lambda_{2} Z_{0}+\theta_{2}\left(Z_{1}\right)\right)
\end{array}\right.
$$

and 


$$
\left\{\begin{aligned}
U^{(1)}= & \alpha_{0} \hat{\gamma}+\left(2 \alpha_{1} \cos \left(2 \lambda_{1} Z_{0}\right)+\alpha_{5}\right) R_{1}^{2}+\left(2 \alpha_{2} \cos \left(2 \lambda_{2} Z_{0}\right)+\alpha_{6}\right) R_{2}^{2} \\
& +2\left(\alpha_{3} \cos \left(\left(\lambda_{1}+\lambda_{2}\right) Z_{0}+\phi_{1}+\phi_{2}\right)+\alpha_{4} \cos \left(\left(-\lambda_{1}+\lambda_{2}\right) Z_{0}-\phi_{1}+\phi_{2}\right)\right) R_{1} R_{2}, \\
G^{(1)}= & \beta_{0} \hat{\gamma}+\left(2 \beta_{1} \cos \left(2 \lambda_{1} Z_{0}+2 \theta_{1}\left(Z_{1}\right)\right)+\beta_{5}\right) R_{1}^{2}+\left(2 \beta_{2} \cos \left(2 \lambda_{2} Z_{0}+2 \theta_{2}\left(Z_{1}\right)\right)+\beta_{6}\right) R_{2}^{2} \\
& +2\left(\beta_{3} \cos \left(\left(\lambda_{1}+\lambda_{2}\right) Z_{0}+\theta_{1}\left(Z_{1}\right)+\theta_{2}\left(Z_{1}\right)\right)\right. \\
& \left.+\beta_{4} \cos \left(\left(\lambda_{1}-\lambda_{2}\right) Z_{0}+\theta_{1}\left(Z_{1}\right)-\theta_{2}\left(Z_{1}\right)\right)\right) R_{1} R_{2} .
\end{aligned}\right.
$$

In the above obtained solutions, there are four unknown constants $R_{1}, R_{2}, \phi_{1}$ and $\phi_{2}$, which can be determined by the end boundary conditions.

Obviously, at $O(1)$ the end boundary conditions (5.3) are automatically satisfied. At $O(\zeta)$, we need

$$
U_{Z_{0}}^{(0)}(0,0)=0, \quad G_{Z_{0}}^{(0)}(0,0)=0, \quad U_{Z_{0}}^{(0)}\left(1, \zeta^{2}\right)=0, \quad G_{Z_{0}}^{(0)}\left(1, \zeta^{2}\right)=0 .
$$

To find the nontrivial solutions, we require that at least one of $R_{1}$ and $R_{2}$ is non-zero; in other words, we will discuss the following two cases: (1) one of $R_{1}$ and $R_{2}$ is zero; (2) both $R_{1}$ and $R_{2}$ are non-zero.

\section{I. $R_{1} \neq 0$ and $R_{2}=0$}

In this subsection, we discuss the case where one of $R_{1}$ and $R_{2}$ equals zero. Without loss of generality, we let $R_{1} \neq 0$ and $R_{2}=0$. For this case, there are two unknown constants $R_{1}$ and $\phi_{1}$, which can be determined by the end conditions (6.17). To satisfy (6.17), from (6.15) and (6.13), we have

$$
\left\{\begin{array}{l}
\sin \phi_{1}=0 \\
f\left(\lambda_{1}\right) \sin \phi_{1}=0 \\
\sin \left(\lambda_{1}+\frac{1}{s_{3}}\left(s_{0} \hat{\gamma}+s_{1} R_{1}^{2}\right) \zeta^{2}+\phi_{1}\right)=0 \\
f\left(\lambda_{1}\right) \sin \left(\lambda_{1}+\frac{1}{s_{3}}\left(s_{0} \hat{\gamma}+s_{1} R_{1}^{2}\right) \zeta^{2}+\phi_{1}\right)=0 .
\end{array}\right.
$$

We note that when $(6.18)_{1}$ is satisfied, $(6.18)_{2}$ is automatically satisfied. From $(6.18)_{1}$, we have

$$
\phi_{1}=m_{1} \pi \quad \text { with } \quad m_{1}=0, \pm 1, \pm 2, \ldots
$$

Also, when $(6.18)_{3}$ is satisfied, $(6.18)_{4}$ is automatically satisfied. We note that $(6.18)_{3}$ should be satisfied for any $\hat{\gamma}$, and in particular we take $\hat{\gamma} \rightarrow 0$ (which implies that $R_{1} \rightarrow 0$ ) to obtain $\sin \left(\lambda_{1}+\phi_{1}\right)=0$ which yields that

$$
\lambda_{1}=n_{1} \pi \quad \text { with } \quad n_{1}=1,2, \ldots
$$

(we have imposed that $\lambda_{1}>0$ ). Substituting (6.19) and (6.20) into (6.18) $)_{3}$, we obtain

$$
R_{1}^{2}=r_{1} \hat{\gamma}
$$

where $r_{1}=-s_{0} / s_{1}$. This amplitude equation for $R_{1}$ can also be obtained by substituting $\theta_{1}=\phi_{1}$ and $R_{2}=0$ into (6.12).

We recall that $\lambda_{1} \mathrm{i}=n_{1} \pi \mathrm{i}$ is a root of the characteristic equation of (6.4) (i.e. it satisfies (5.12)). Letting $\lambda=n \pi \mathrm{i}$ in (5.12), we recover (5.22), which can be used to determine the relationship between the critical stress value and the mode number. Thus, the nonlinear bifurcation analysis presented in this section can recover the results obtained by the linear bifurcation analysis.

Substituting the expressions of $\theta_{1}, \phi_{1}, \lambda_{1}, R_{1}$ into (6.15) and (6.16), we obtain

$$
\left\{\begin{array}{l}
U \approx U_{h}+2(-1)^{m_{1}}\left(r_{1} \Delta \gamma\right)^{1 / 2} \cos \left(n_{1} \pi Z\right)+\left(\alpha_{0}+\left(2 \alpha_{1} \cos \left(2 n_{1} \pi Z\right)+\alpha_{5}\right) r_{1}\right) \Delta \gamma \\
G \approx G_{h}+2 f\left(n_{1} \pi\right)(-1)^{m_{1}}\left(r_{1} \Delta \gamma\right)^{1 / 2} \cos \left(n_{1} \pi Z\right)+\left(\beta_{0}+\left(2 \beta_{1} \cos \left(2 n_{1} \pi Z\right)+\beta_{5}\right) r_{1}\right) \Delta \gamma
\end{array}\right.
$$


which are the analytical expressions for describing the post-bifurcation states when $\Delta \gamma$ is small.

Similarly, when $R_{1}=0$ and $R_{2} \neq 0$, the approximate solutions of $U$ and $G$ are given by

$$
\left\{\begin{array}{l}
U \approx U_{h}+2(-1)^{m_{2}}\left(r_{2} \Delta \gamma\right)^{1 / 2} \cos \left(n_{2} \pi Z\right)+\left(\alpha_{0}+\left(2 \alpha_{1} \cos \left(2 n_{2} \pi Z\right)+\alpha_{6}\right) r_{2}\right) \Delta \gamma, \\
G \approx G_{h}+2 f\left(n_{2} \pi\right)(-1)^{m_{2}}\left(r_{2} \Delta \gamma\right)^{1 / 2} \cos \left(n_{2} \pi Z\right)+\left(\beta_{0}+\left(2 \beta_{2} \cos \left(2 n_{2} \pi Z\right)+\beta_{6}\right) r_{2}\right) \Delta \gamma,
\end{array}\right.
$$

where $r_{2}=-t_{0} / t_{1}, m_{2}=0, \pm 1, \pm 2, \ldots$ and $n_{2}=1,2, \ldots$

The simple analytical expressions (6.22) and (6.23) can provide both qualitative and quantitative information on the post-bifurcation states (in general, it is very difficult, if even possible, to deduce qualitative information from numerical solutions). Now, we give some discussions. It should be noted that the information below cannot be deduced from a linear bifurcation analysis.

First, from the expression (6.21) for the amplitude, we see that the sign of $r_{1}$, which depends on $U_{h}$, $G_{h}, \rho$ and $\nu_{0}$, determines the nature of the bifurcations. If $r_{1}<0$, we need $\Delta \gamma=\zeta^{2} \hat{\gamma}<0$ to ensure the positivity of $R_{1}^{2}$. Thus the bifurcation occurs only when $|\gamma|>\left|\gamma_{c}\right|$, which is a supercritical bifurcation. Conversely, if $r_{1}>0$, we need $\Delta \gamma=\zeta^{2} \hat{\gamma}>0$ and the bifurcation occurs only when $|\gamma|<\left|\gamma_{c}\right|$, which is a subcritical bifurcation. For the geometrical parameters considered here, we find that $r_{1}$ is always negative, and thus all bifurcations in the case of $R_{1} \neq 0$ and $R_{2}=0$ are supercritical.

Also, in the present case we see that the solutions (6.22) and (6.23) contain $m_{1}$ or $m_{2}$. Since $m_{1}$ and $m_{2}$ can be even or odd, there are two nontrivial solutions for a given mode number. This implies that it is a supercritical pitchfork bifurcation (see Figure 12).

It is expected that the geometrical parameters have important influences on the post-bifurcation states. For the energy absorption purpose, usually one would like the structure to be as 'soft' as possible in the post-bifurcation regime. Here, we say that a structure is 'soft' if for a reduction of the length of the tube the end stress is small. The usual thinking is that a thin structure is softer than a thick structure. However, it may not be true in the post-bifurcation regime. As we shall see below, increasing the thickness of the tube slightly can reduce the stress significantly.

We denote the leading-order amplitudes of $U$ and $G$ as $d_{1}$ and $d_{2}$ respectively. It can be seen from (6.22) and (6.23) that $d_{1}$ can be described through $2\left|r_{1}\right|^{1 / 2}$ or $2\left|r_{2}\right|^{1 / 2}$ and $d_{2}$ through $2 f\left(n_{1} \pi\right)\left|r_{1}\right|^{1 / 2}$ or $2 f\left(n_{2} \pi\right)\left|r_{2}\right|^{1 / 2}$ (which are dependent on the geometrical parameters $\rho$ and $\nu_{0}$ and the mode number; please note that $U_{h}$ and $G_{h}$ are dependent on $\gamma_{c}$, which, in turn, is determined by $\rho$ and $\nu_{0}$ and the mode number (see (5.22)).

In Figure 6, we plot the curves of $d_{1}$ and $d_{2}$ for $\nu_{0}=0.1$ (corresponding to Figure 3(a)). Please note that for $\rho^{(k+1, k+2)}<\rho<\rho^{(k, k+1)}\left(k=3, \ldots, 8\right.$ for $\left.\nu_{0}=0.1\right)$ the first mode number is $k$ (see the discussions above (5.23)). As a result, we only draw the curves corresponding to the $k$ th mode for $\rho$ in this interval. From this figure, we find that both $d_{1}$ and $d_{2}$ are increasing functions of $\rho$ for $\rho^{(k+1, k+2)}<\rho$ $<\rho^{(k, k+1)}$. Then, corresponding to the same mode number, it is expected that as the engineering stress increases the current length of the thicker tube after bifurcation will decrease faster than that of the thinner tube.

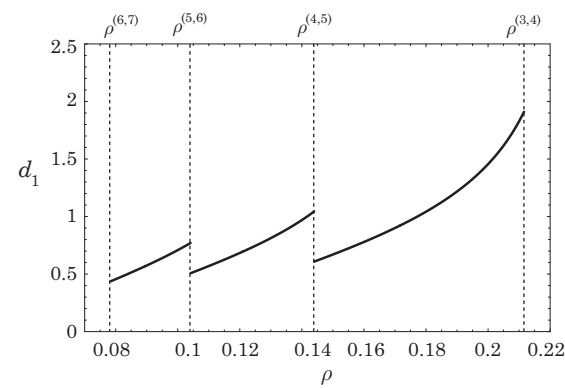

(a) $d_{1}$ vs $\rho$

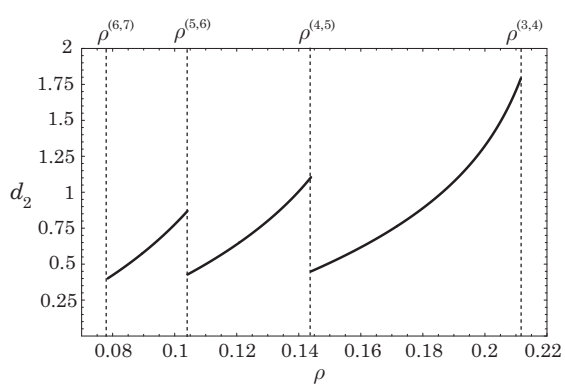

(b) $d_{2}$ vs $\rho$

Figure 6. Plots of $d_{1}$ and $d_{2}$ vs $\rho$ for $\nu_{0}=0.1$. 


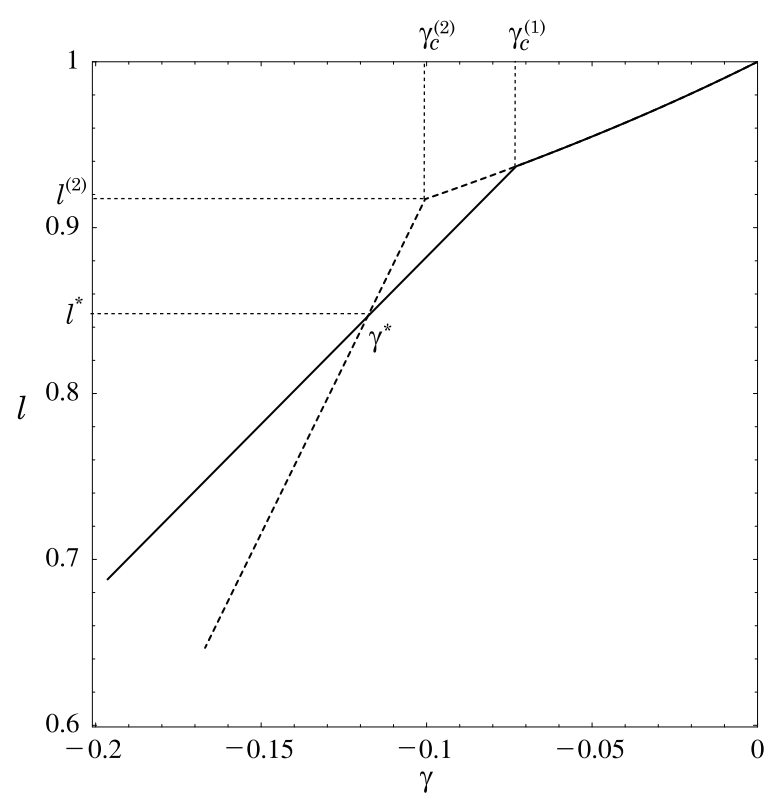

Figure 7. Plot of the length of the tube $I$ vs the engineering stress $\gamma$ when $\nu_{0}=0.1$ (full lines are for $\rho=0.105$ and dashed lines are for $\rho=0.135)$.

In Figure 7, we give the plots of the current length $l$ of the tube after bifurcation versus the engineering stress $\gamma$ for two slightly different tube thicknesses (however, the first bifurcation mode is still the same). In Figure 7, curves are plotted for $\rho=0.105$ and $\rho=0.135$ both of which give the same first bifurcation mode $n=5$ as we can see from Figure 6(a). We note that the difference between the two thicknesses is about $15 \%$. The thinner tube will bulge first at a smaller critical stress $\left|\gamma_{c}^{(1)}\right|$ than that of the thicker tube (i.e. $\left.\left|\gamma_{c}^{(2)}\right|\right)$ as expected. After the bifurcations, the two curves intersect at $\left(\gamma^{*}, l^{*}\right)$. We observe that when $\left|\gamma_{c}^{(1)}\right|<|\gamma|<\left|\gamma^{*}\right|$, the engineering stress of the thicker tube is larger than that of the thinner tube for the same length of the tube, so the thinner tube is 'softer'. When $|\gamma|>\left|\gamma^{*}\right|$, for the same tube length (or the same reduction of its original length) the engineering stress in the thicker tube is significantly smaller than that in the thinner tube. The difference in stress can be large when $l$ is getting smaller. Thus, in this post-bifurcation regime the thicker tube is softer than the thinner tube. We can see that the geometry (thickness) of the tube can affect the softness of the tube greatly, and this effect may be useful in designing a tube as an energy absorber.

\section{Remarks:}

1. For this case, the amplitude equation obtained from (6.12) is

$$
s_{0} \hat{\gamma} R_{1}+s_{1} R_{1}^{3}=0 .
$$

For this kind of amplitude equation, it has been shown by the singularities theory in [32] that for a supercritical bifurcation the structure is imperfection-insensitive and for a subcritical bifurcation it is imperfection-sensitive. Since we have a supercritical pitchfork bifurcation here, the tube is imperfection-insensitive in the case discussed in this section.

2. Since there are multiple solutions when the external stress exceeds the critical stress value, we need to calculate the total elastic potential energy to determine which solution has the smallest value, that is, which is the energetically preferred solution. For the current forcecontrolled problem, the asymptotic expression of the total elastic potential energy of the tube is given by 


$$
\begin{aligned}
J= & \pi \delta \mu_{0} \int_{0}^{1}\left(\frac{4}{3} U^{2}-\frac{52}{27} U^{3}+\frac{199}{81} U^{4}+G\left(\frac{2}{3} U-\frac{1}{9} U^{2}+\frac{4}{81} U^{3}\right)\right. \\
& +G^{2}\left(\frac{4}{3}-\frac{1}{9} U+\frac{1}{54} U^{2}\right)+G^{3}\left(-\frac{52}{27}+\frac{4 U}{81}\right)+\frac{199}{81} G^{4}+\nu_{0}\left(\frac{1}{3} U U_{Z}^{2}+\frac{4}{3} U_{Z}^{2} G\right) \\
& +\delta\left(-\frac{5}{9} U U_{Z}^{2}-\frac{1}{6} U U_{Z Z}+\frac{1}{12} U^{2} U_{Z Z}+G\left(-\frac{5}{36} U_{Z}^{2}-\frac{2}{3} U_{Z Z}+\frac{1}{2} U U_{Z Z}\right)\right. \\
& +\frac{7}{3} U_{Z Z} G^{2}+\frac{1}{6} U U_{Z} G_{Z}+\frac{2}{3} U_{Z} G G_{Z}+\frac{1}{\nu_{0}}\left(-\frac{8}{9} U^{2}+\frac{56}{27} U^{3}-\frac{902}{243} U^{4}\right. \\
& +G\left(-\frac{4}{9} U+\frac{2}{3} U^{2}-\frac{272}{243} U^{3}\right)+G^{2}\left(-\frac{1}{18}+\frac{2}{9} U-\frac{34}{81} U^{2}\right) \\
& \left.\left.\left.+G^{3}\left(\frac{5}{108}-\frac{79}{486} U\right)-\frac{19}{486} G^{4}\right)\right)\right) d Z-\frac{5}{2} \pi \delta \mu_{0} \gamma \int_{0}^{1} G d Z
\end{aligned}
$$

By using (6.24), the approximate analytical solutions (6.22) and (6.23) for the post-bifurcation solutions and the algebraic equations (5.5a) and (5.5b) for the trivial solutions, we can calculate the total potential energy values for nontrivial solutions and the corresponding trivial solutions. For some parameter values, the results are listed in Table 2.

In Table 2 we find that for each case two nontrivial solutions have the same energy value, which is less than that of the trivial solution. Then two nontrivial solutions are both the preferred solution. An unfortunate side is that the present theory is unable to determine whether the solution with $m_{1}=1$ or that with $m_{1}=2$ is more preferred, although the solution profiles are very different.

\section{2. $R_{l} \neq 0$ and $R_{2} \neq 0$}

In this subsection, we discuss the case where both $R_{1}$ and $R_{2}$ do not equal zero. For this case, there are four unknown constants $R_{1}, R_{2}, \phi_{1}$ and $\phi_{2}$, and they can also be determined by the end conditions (6.17). By using an analysis similar to that in the above section, we can obtain

$$
\begin{aligned}
& \phi_{1}=m_{1} \pi, \quad \phi_{2}=m_{2} \pi \quad \text { with } \quad m_{1}, m_{2}=0, \pm 1, \pm 2, \ldots, \\
& \lambda_{1}=n_{1} \pi, \quad \lambda_{2}=n_{2} \pi \quad \text { with } \quad n_{1}, n_{2}=1,2, \ldots\left(n_{1} \neq n_{2}\right)
\end{aligned}
$$

and

$$
s_{0} \hat{\gamma}+s_{1} R_{1}^{2}+s_{2} R_{2}^{2}=0, \quad t_{0} \hat{\gamma}+t_{1} R_{2}^{2}+t_{2} R_{1}^{2}=0 .
$$

From (6.13), (6.14) and (6.26), we have $\theta_{1}=\phi_{1}$ and $\theta_{2}=\phi_{2}$.

Solving the amplitudes equations (6.26), we have

$$
R_{1}^{2}=r_{1} \hat{\gamma}, \quad R_{2}^{2}=r_{2} \hat{\gamma},
$$

where $r_{1}=\left(t_{0} s_{2}-t_{1} s_{0}\right) /\left(s_{1} t_{1}-s_{2} t_{2}\right)$ and $r_{2}=\left(s_{0} t_{2}-s_{1} t_{0}\right) /\left(s_{1} t_{1}-s_{2} t_{2}\right)$.

We note that both $\lambda_{1} \mathrm{i}=n_{1} \pi \mathrm{i}$ and $\lambda_{2} \mathrm{i}=n_{2} \pi \mathrm{i}\left(n_{1} \neq n_{2}\right)$ should satisfy the characteristic equation of (6.4) simultaneously. That is, both $n_{1}$ and $n_{2}$ satisfy (5.22). Thus, when both $R_{1} \neq 0$ and $R_{2} \neq 0$, there

Table 2. Total potential energy values scaled by $\pi \delta \mu_{0}$.

\begin{tabular}{lll}
\hline & Nontrivial solutions & Trivial solution \\
\hline (I) $\nu_{0}=0.1, \rho=0.16, \gamma=-0.13$ & $-0.0211568\left(m_{1}=1,2\right)$ & -0.0178934 \\
(2) $\nu_{0}=0.1, \rho=0.13, \gamma=-0.096$ & $-0.0112424\left(m_{2}=1,2\right)$ & -0.010141 \\
(3) $\nu_{0}=0.1, \rho=0.1, \gamma=-0.07$ & $-0.0075429\left(m_{2}=1,2\right)$ & -0.0055647 \\
\hline
\end{tabular}


are two different mode numbers corresponding to the same critical stress value. It should be noted that this case is a special one, as for it to happen there is a relation between the two geometrical parameters $\nu_{0}$ and $\rho$ (since both $n_{1}$ and $n_{2}$ satisfy (5.22)).

After substituting the expressions of $\theta_{i}, \phi_{i}, \lambda_{i}$ and $R_{i}(i=1,2)$ into (6.15) and (6.16), we obtain

$$
\begin{gathered}
U \approx U_{h}+2(-1)^{m_{1}}\left(r_{1} \Delta \gamma\right)^{1 / 2} \cos \left(n_{1} \pi Z\right)+2(-1)^{m_{2}}\left(r_{2} \Delta \gamma\right)^{1 / 2} \cos \left(n_{2} \pi Z\right) \\
+\left(\alpha_{0}+\left(2 \alpha_{1} \cos \left(2 n_{1} \pi Z\right)+\alpha_{5}\right) r_{1}+\left(2 \alpha_{2} \cos \left(2 n_{2} \pi Z\right)+\alpha_{6}\right) r_{2}\right) \Delta \gamma \\
-2(-1)^{m_{1}+m_{2}}\left(\alpha_{3} \cos \left(\left(n_{1}+n_{2}\right) \pi Z\right)+\alpha_{4} \cos \left(\left(n_{1}-n_{2}\right) \pi Z\right)\right)\left(r_{1} r_{2}\right)^{1 / 2} \Delta \gamma, \\
G \approx G_{h}+2 f\left(n_{1} \pi\right)(-1)^{m_{1}}\left(r_{1} \Delta \gamma\right)^{1 / 2} \cos \left(n_{1} \pi Z\right)+2 f\left(n_{2} \pi\right)(-1)^{m_{2}}\left(r_{2} \Delta \gamma\right)^{1 / 2} \cos \left(n_{2} \pi Z\right) \\
+\left(\beta_{0}+\left(2 \beta_{1} \cos \left(2 n_{1} \pi Z\right)+\beta_{5}\right) r_{1}+\left(2 \beta_{2} \cos \left(2 n_{2} \pi Z\right)+\beta_{6}\right) r_{2}\right) \Delta \gamma \\
-2(-1)^{m_{1}+m_{2}}\left(\beta_{3} \cos \left(\left(n_{1}+n_{2}\right) \pi Z\right)+\beta_{4} \cos \left(\left(n_{1}-n_{2}\right) \pi Z\right)\right)\left(r_{1} r_{2}\right)^{1 / 2} \Delta \gamma,
\end{gathered}
$$

which are the analytical solutions for describing four $\left(m_{1}=1,2\right.$ and $\left.m_{2}=1,2\right)$ post-bifurcation states for small $\Delta \gamma$ when both $n_{1}$ and $n_{2}$ satisfy (5.22).

Now, we discuss some bifurcation features for this case.

First, from (6.27) we see that we need both $r_{1}<0$ and $r_{2}<0$ or both $r_{1}>0$ and $r_{2}>0$, which correspond to a supercritical bifurcation and a subcritical bifurcation, respectively. For the present problem we find that within the imposed constraint on the geometrical parameters we always have $r_{1}>0$ and $r_{2}>0$. Thus, those solutions given in (6.28) represent subcritical branches.

Also, we note that when $n_{1}$ and $n_{2}$ satisfy (5.22) there are also another four post-bifurcation states corresponding to $R_{1} \neq 0$ and $R_{2}=0$, and $R_{1}=0$ and $R_{2} \neq 0$, since (6.17) is also satisfied for those cases. The solution expressions are provided by (6.22) $\left(m_{1}=1,2\right)$ and $(6.23)\left(m_{2}=1,2\right)$. Each of these four branches is supercritical, as discussed in the previous section. Thus, in the case of two different modes corresponding to the same critical stress there are in total eight post-bifurcation states around the bifurcation point, four subcritical branches and four supercritical branches. We call such a bifurcation an octopus bifurcation (see Figure 15).

The study on the imperfection sensitivity for this case, which needs certain tools in singularities theory, is presented in the next section.

Remark: In obtaining the analytical solutions for the above two different cases, we only use the sliding boundary conditions at $O(\zeta)$. It is easy to verify that the obtained solutions also satisfy the conditions at $O\left(\zeta^{2}\right)$.

\section{Imperfection sensitivity for the case of two modes corresponding to a critical stress}

Subcritical bifurcation branches occur when two modes correspond to the same critical stress value. The important feature of a subcritical bifurcation is the imperfection sensitivity. In this section, we will use singularities theory to study this important issue for this case. We take $\nu_{0}=0.1$ and $\rho=0.103995$ for example (correspondingly the two modes are $n_{1}=5, n_{2}=6$ and the critical stress is $\gamma_{c}=-0.0722086$ ) and follow the discussions in [22, 23].

In Section 6 we obtained the amplitudes equations (6.21) when $R_{1} \neq 0$ and $R_{2}=0$ and (6.26) when $R_{1} \neq 0$ and $R_{2} \neq 0$. The amplitudes equations, which include both the trivial solutions and the nontrivial solutions of $R_{1}$ and $R_{2}$, are given by (see (6.12))

$$
s_{0} \hat{\gamma} R_{1}+s_{1} R_{1}^{3}+s_{2} R_{1} R_{2}^{2}=0, \quad t_{0} \hat{\gamma} R_{2}+t_{1} R_{2}^{3}+t_{2} R_{2} R_{1}^{2}=0 .
$$

For the parameters chosen above, we find that the non-degeneracy conditions defined in Definition 2.2 [23, p. 423] are satisfied. Then according to Proposition 2.3 in [23, p. 424], the bifurcation problem of (7.1) with two state variables has the normal form

$$
\varepsilon_{1} R_{1}^{3}+p R_{1} R_{2}^{2}+\varepsilon_{2}(-\hat{\gamma}) R_{1}=0, \quad q R_{2} R_{1}^{2}+\varepsilon_{3} R_{2}^{3}+\varepsilon_{4}(-\hat{\gamma}) R_{2}=0,
$$

where 


$$
\begin{aligned}
& \varepsilon_{1}=\operatorname{sgn}\left(s_{1}\right), \quad \varepsilon_{2}=\operatorname{sgn}\left(-s_{0}\right), \quad \varepsilon_{3}=\operatorname{sgn}\left(t_{1}\right), \quad \varepsilon_{4}=\operatorname{sgn}\left(-t_{0}\right), \\
& p=\left|\frac{t_{0}}{t_{1} s_{0}}\right| s_{2}, \quad q=\left|\frac{s_{0}}{s_{1} t_{0}}\right| t_{2}, \quad p \neq \varepsilon_{2} \varepsilon_{3} \varepsilon_{4}, \quad q \neq \varepsilon_{1} \varepsilon_{2} \varepsilon_{4}, \quad p q \neq \varepsilon_{1} \varepsilon_{3} .
\end{aligned}
$$

From Theorem 2.4 in [23, p. 425], the normal form (7.2) has the following universal unfolding:

$$
\varepsilon_{1} R_{1}^{3}+p R_{1} R_{2}^{2}+\varepsilon_{2}(-\hat{\gamma}) R_{1}=0, \quad q R_{2} R_{1}^{2}+\varepsilon_{3} R_{2}^{3}+\varepsilon_{4}(-\hat{\gamma}-\sigma) R_{2}=0,
$$

where $\sigma$ is a small parameter representing the imperfection magnitude.

For the chosen $\rho$ and $\nu_{0}$, we find that

$$
\varepsilon_{1}=\varepsilon_{3}=1, \quad \varepsilon_{2}=\varepsilon_{4}=-1 .
$$

For the above case, a detailed discussion on the bifurcation diagrams with different values of $p$ and $q$ has been given in [23]. For $\nu_{0}=0.1$ and $\rho=0.103995$, we find that $p=-15.12$ and $q=-4.71$, and the corresponding unperturbed and perturbed bifurcation diagrams are given in Figures 8 and 9. The solid lines represent the stable solutions and the dashed lines represent the unstable solutions.

From Figure 9, we find that when $\sigma>0$, the real critical stress value is not changed, and so this value is not imperfection-sensitive. In this case, the stable branch is the supercritical $R_{1}$-mode. However, with a further increase of the load (an $O(\sigma)$ amount), this mode also becomes unstable. Then, the tube has to jump to a state corresponding to a remote stable solution (which cannot be determined by the present theory). In this sense, we may say that the structure is imperfection-sensitive.

When $\sigma<0$, the real critical stress value becomes

$$
\gamma^{*}=\gamma_{c}-\sigma,
$$

whose absolute value is smaller than that of the unperturbed critical stress value. So, this value is imperfection-sensitive. Afterwards, when the load increases, the solution goes along the supercritical $R_{2}$-mode. However, with a further increase of the load (an $O(\sigma)$ amount), this branch becomes unstable. Then, the tube has to jump to a state corresponding to a remote stable solution (which cannot be determined by the present theory). Thus, the tube is imperfection-sensitive.

As discussed in Section 5, for a thin tube it very easily happens that two modes correspond to the same critical stress. The theory presented here gives the explanation as to why a thin tube is usually imperfection-sensitive. For a thick tube, only if the two geometrical parameters $\rho$ and $\nu_{0}$ satisfy a

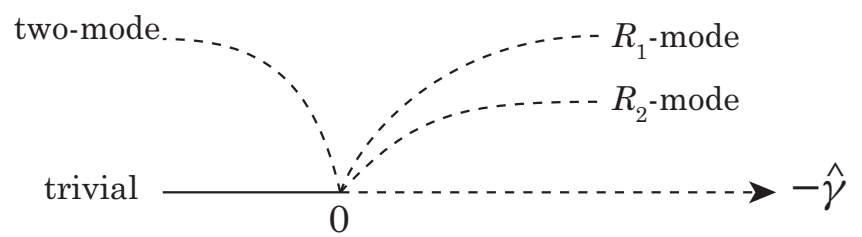

Figure 8. Unperturbed schematic bifurcation diagram.

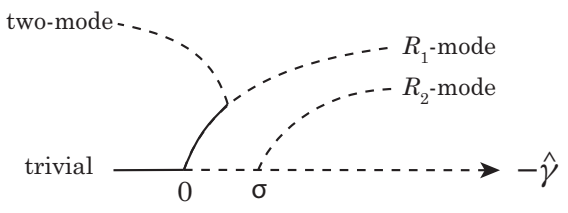

(a) $\sigma>0$

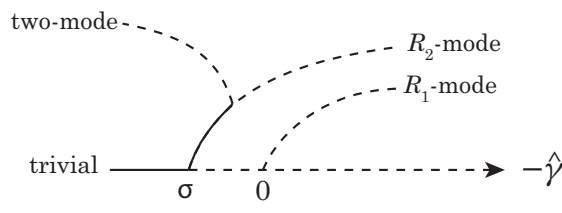

(b) $\sigma<0$

Figure 9. Perturbed schematic bifurcation diagrams. 
further relation are there two modes corresponding to the same critical stress. Then, in general, a thick tube is imperfection-insensitive.

We remark that imperfection sensitivity may not be a bad thing when the tube is used for energy absorption purposes, since, corresponding to the remote solutions mentioned above, the structure may be softer. Of course, one has to examine the remote solutions to draw such a conclusion, which will be left for future investigation.

\section{Numerical solutions and graphic results}

In Sections 5 and 6, we have determined the critical stress values of $\gamma_{c}$ analytically and obtained the analytical post-bifurcation solutions by the multiple scales method. To assess the validity of the analytical results, in this section we use the bifurcation analysis software AUTO (see [33]) to do numerical experiments, and comparisons of the results obtained by two approaches will be made. Some graphic results will also be presented.

It is relatively straightforward to use AUTO to find the bifurcation points of a system of ODEs with specific boundary conditions, which can then yield the critical stress values for the present problem. Some critical stress values $\gamma_{c}$ for the first mode number obtained in Section 5 are compared with those obtained by AUTO in Table 3. We find that the values obtained from the analytical expressions are very close to the numerical values (the largest relative error among three cases is about $3 \%$ ). Thus, (5.22) provides reliable results for the critical stress values.

To use AUTO to compute post-bifurcation solutions of boundary-value problems could be a nontrivial matter. In fact, we intended to get the numerical solutions by AUTO first and then use them to help us construct the analytical solutions. However, the outcome was not satisfactory since we were not sure whether AUTO missed a branch of solutions. It turned out the other way around. We obtained the analytical solutions first to find all the bifurcated branches, which then helped us define the proper amplitude of the solutions in AUTO programs. And finally, AUTO can yield all the post-bifurcation solutions. The numerical solutions of the third case in Table 3 are plotted in Figure 10.

For the cases listed in Table 3, there is only one mode number corresponding to a critical stress value, and the analytical solutions of $U$ and $G$ are given by (6.22) when $R_{2}=0$ and (6.23) when $R_{1}=0$. The solution curves of the third case in Table 3 are plotted in Figure 10. One can see that there is very good agreement between the numerical solutions obtained by AUTO and the analytical solutions.

Table 3. Comparisons of the critical stress values.

\begin{tabular}{llll}
\hline & $n$ & $\gamma_{c}$ (Numerical) & $\gamma_{c}($ Analytical) \\
\hline (I) $\nu_{0}=0.1, \rho=0.16$ & 4 & -0.125478 & -0.121826 \\
$(2) \nu_{0}=0.1, \rho=0.13$ & 5 & -0.0948662 & -0.0935275 \\
(3) $\nu_{0}=0.1, \rho=0.1$ & 6 & -0.0680846 & -0.06772 \\
\hline
\end{tabular}

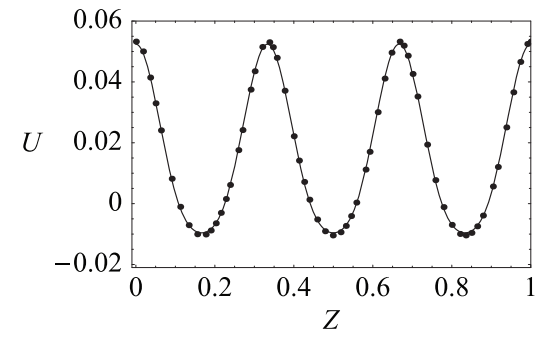

(a) $m_{2}=2$

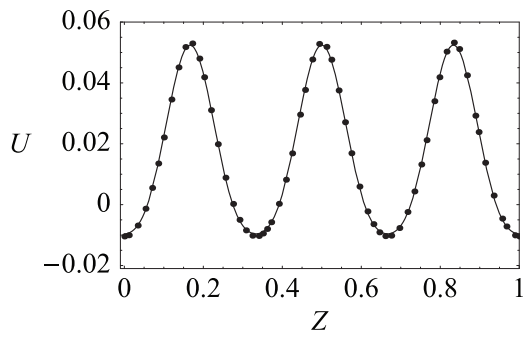

(b) $m_{2}=1$

Figure 10. Comparisons of the analytical solutions (solid curves) of $U$ with the numerical solutions (dots) for $\nu_{0}=0.1, \rho=0.1$, $n_{2}=6$ and $\gamma=-0.07$. 


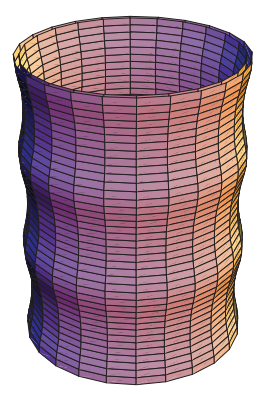

(a) $m_{2}=2$

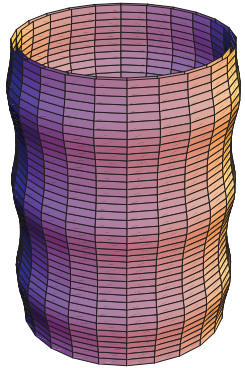

(b) $m_{2}=1$

Figure I I. Shapes of the buckled tubes corresponding to the case in Figure 10.

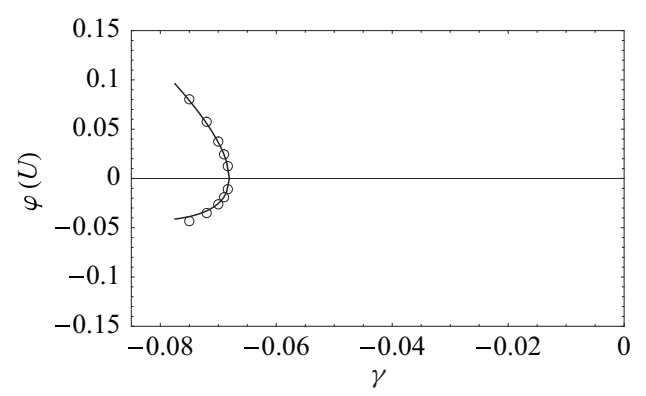

Figure 12. Pitchfork bifurcation diagrams for $\nu_{0}=0.1$ and $\rho=0.1$. Full lines are the analytical solutions and circles are the numerical solutions.

Table 4. The critical stress values when two modes exist.

\begin{tabular}{lll}
\hline & $n$ & $\gamma_{c}$ \\
\hline$(1) \nu_{0}=0.1, \rho=0.103995$ & $n_{1}=5, n_{2}=6$ & -0.0722086 \\
$(2) \nu_{0}=0.22, \rho=0.115096$ & $n_{1}=3, n_{2}=4$ & -0.0847745 \\
\hline
\end{tabular}

By using (2.1) and the analytical expressions of $U$ and $G$, we can plot the three-dimensional shapes of the buckled tubes in the current configuration. The buckled shapes corresponding to the cases in Figure 10 are given in Figure 11.

For given $\nu_{0}, \rho$ and mode numbers $n$ listed in Table 3, we find that these bifurcations are pitchfork and supercritical bifurcations. This type of bifurcation is illustrated in Figure 12, which gives the bifurcation diagrams for case (3) in Table 3. In this figure, the bifurcation diagrams plotted by using the analytical solutions of $U$ are compared with the numerical results obtained by AUTO and the amplitude $\varphi(U)$ is defined by

$$
\varphi(U)=U(0)-U_{h} .
$$

Once again, very good agreement between the analytical solutions and the numerical ones is found.

Those comparisons demonstrate that the simple analytical expressions (6.22) and (6.23) provide reliable results for the post-bifurcation states. We note that when we further increase the external stress it is found that the tube intends to deform with folds.

As we discussed for Figure 3 in Section 5, for the particular value of $\rho$ at the intersection point, two modes correspond to the same critical stress value. Two examples are listed in Table 4. 


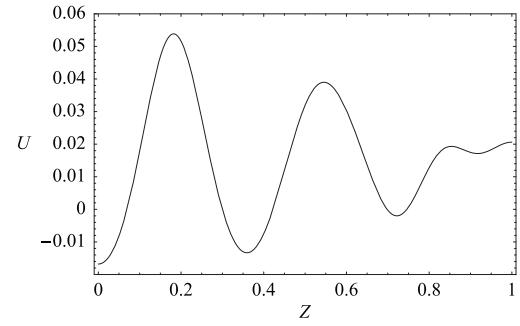

(a) $m_{1}=1$ and $m_{2}=1$

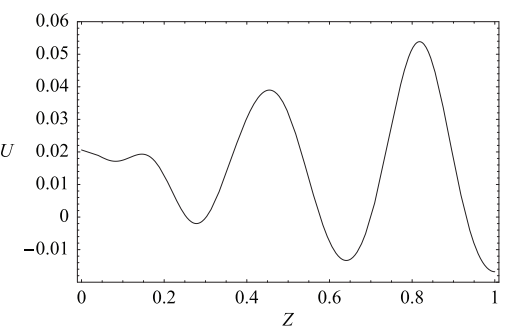

(c) $m_{1}=2$ and $m_{2}=1$

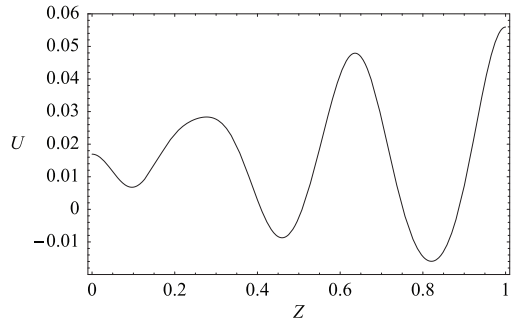

(b) $m_{1}=1$ and $m_{2}=2$

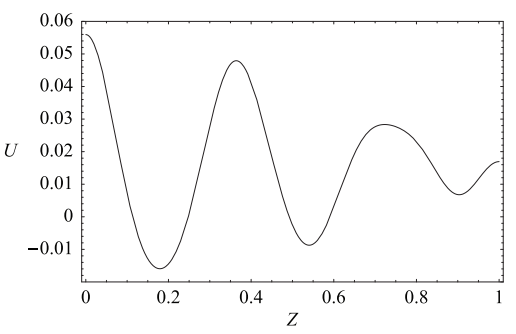

(d) $m_{1}=2$ and $m_{2}=2$

Figure 13. Plots of the approximate analytical solutions of $U$ when $\nu_{0}=0.1, \gamma=-0.066$ and $\rho=0.103995\left(\gamma_{c}=-0.0722086\right.$, $n_{1}=5$ and $\left.n_{2}=6\right)$.

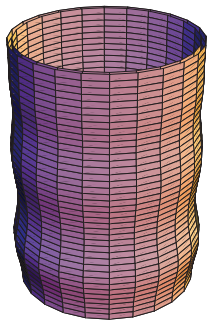

(a) $m_{1}=1, m_{2}=1$

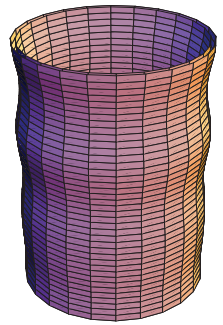

(b) $m_{1}=1, m_{2}=2$

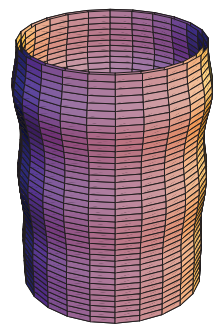

(c) $m_{1}=2, m_{2}=1$

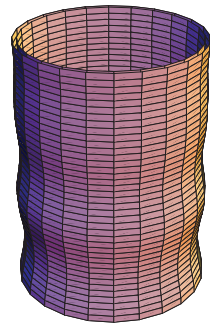

(d) $m_{1}=2, m_{2}=2$

Figure 14. Shapes of the buckled tubes corresponding to the cases in Figure 13 when $\gamma=-0.066$.

For the two cases in Table 4, there are two different modes $n_{1}$ and $n_{2}$ corresponding to the same critical stress value. Unfortunately, AUTO fails to get the bifurcation point and any branch of solutions for this case (on the other hand, the failure of the numerical approach demonstrates the indispensability of analytical solutions). Therefore, in the following we shall present some graphic results based on the analytical solutions.

According to the analytical results in Section 6, there exist eight nontrivial solution branches for the particular $\rho$ and $\nu_{0}$ when there are two different modes corresponding to the same critical stress value (two branches corresponding to the unique mode $n_{1}$, two branches corresponding to the unique mode $n_{2}$ and four branches corresponding to two modes $n_{1}$ and $n_{2}$ ). In Figure 13, we plot the four subcritical post-bifurcation solutions of $U$ by using the corresponding approximate analytical solutions.

Also, by using (2.1) and the analytical expressions of $U$ and $G$, the shapes of the buckled tubes corresponding to the cases in Figure 13 are given in Figure 14.

As discussed before, the bifurcation corresponding to a unique mode number is a supercritical one and the bifurcation corresponding to two mode numbers is a subcritical one. Thus, the supercritical and the subcritical bifurcations can occur simultaneously when two modes correspond to the same critical 


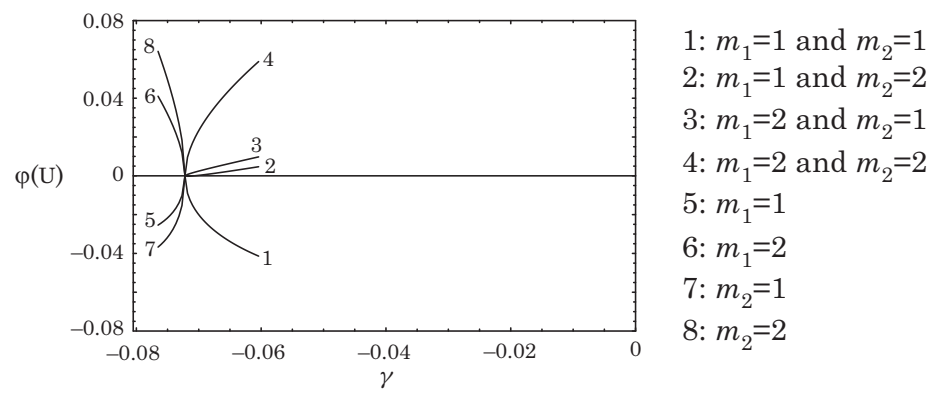

Figure 15. Octopus bifurcation diagram for $\nu_{0}=0.1$ and $\rho=0.103995$.

stress value. The bifurcation diagrams in Figure 15 are plotted by using the approximate analytical solutions of $U$. This type of bifurcation was called an octopus bifurcation at the end of Section 6 since there are eight 'legs' (branches) around the bifurcation point.

\section{Concluding remarks}

Linear and nonlinear bifurcation analysis is conducted for the axisymmetric deformations of a hyperelastic tube subjected to axial compression. The analytical results reveal many fresh insights, including:

(a) The critical stress has a uniform lower bound (independent of the slenderness) for fixed inner and outer radii.

(b) For the single-mode case the bifurcation is a supercritical pitchfork bifurcation.

(c) For the case of two modes corresponding to a critical stress the bifurcation is an octopus bifurcation, which is the mechanism for imperfection sensitivity.

(d) An explanation is given as to why a thin tube is usually imperfection-sensitive while a thick tube is not in general.

(e) In the post-bifurcation regime a thick tube could be considerably softer than a thin one (which may be useful in designing a tube as an energy absorber).

(f) Within the framework of hyperelasticity fold-like profiles can develop in the post-bifurcation regime.

The methodology is systematic without ad hoc assumptions and is based on the three-dimensional field equations of finite deformations (taking into account both geometrical and material nonlinearity). The method of coupled series-asymptotic expansions is adopted to derive the asymptotic model equations in a mathematically consistent manner. Then, the method of multiple scales is used to construct the postbifurcation solutions at near-critical loads. With the obtained amplitude equations, the singularities theory is used to study the imperfection sensitivity. We believe that the approach presented here is general and can be used to study other instability phenomena in structures composed of nonlinearly elastic materials (e.g. the buckling of a thin nonlinearly elastic film on a nonlinearly elastic compliant substrate). We shall leave these for future investigations.

\section{Declaration of conflicting interests}

None declared.

\section{Funding}

This work was supported by a grant from the Research Grants Council of the HKSAR, China (project number CityU 100911), the National Natural Science Foundation of China (project number 11102068) and the Fundamental Research Funds for the Central Universities (project number WM1214042). 


\section{References}

[1] Koiter, WT. On the stability of elastic equilibrium. (Dutch.) Thesis, Polytechnic Institute of Delft, The Netherlands, 1945.

[2] Van der Heijden, AMA. W. T. Koiter's elastic stability of solids and structures. Cambridge: Cambridge University Press, 2009.

[3] Hutchinson, JW. Imperfection sensitivity of externally pressurized spherical shell. J Appl Mech 1967; 34: 49-55.

[4] Hunt, GW, et al. Cylindrical shell buckling: A characterization of localization and periodicity. Discr Contin Dyn Syst Ser B 2003; 3: 505-518.

[5] Beatty, MF. Topics in finite elasticity. Appl Mech Rev 1987; 40: 1699-1734.

[6] Wilkes, EW. On the stability of a circular tube under end thrust. Q J Mech Appl Math 1955; 8: 89-100.

[7] Pan, F, and Beatty, MF. Instability of a Bell constrained cylindrical tube under end thrust. Part 1: Theoretical development. Math Mech Solids 1997; 2: 243-273.

[8] Pan, F, and Beatty, MF. Remarks on the instability of an incompressible and isotropic hyperelastic, thick-walled cylindrical tube. J Elast 1997; 48: 217-239.

[9] Pan, F, and Beatty, MF. Instability of a Bell constrained cylindrical tube under end thrust. Part 2: Examples, thin tube analysis. Math Mech Solids 1999; 4: 227-250.

[10] Haughton, DM, and Ogden, RW. Bifurcation of inflated circular cylinders of elastic material under axial loading - I. Membrane theory for thin-walled tubes. J Mech Phys Solids 1979; 27: 179-212.

[11] Haughton, DM, and Ogden, RW. Bifurcation of inflated circular cylinders of elastic material under axial loading - II. Exact theory for thick-walled tubes. J Mech Phys Solids 1979; 27: 489-512.

[12] Zhu, Y, et al. Asymmetric bifurcations of thick-walled circular cylindrical elastic tubes under axial loading and external pressure. Int J Solids Struct 2008; 45: 3410-3429.

[13] Dorfmann, A, and Haughton, DM. Stability and bifurcation of compressed elastic cylindrical tubes. Int J Eng Sci 2006; 44: $1353-1365$.

[14] Goriely, A, et al. Nonlinear Euler buckling. Proc R Soc Lond A 2008; 464: 3003-3019.

[15] Dai, H-H, and Huo, Y. Asymptotically approximate model equations for nonlinear dispersive waves in incompressible elastic rods. Acta Mech 2002; 157: 97-112.

[16] Dai, H-H, and Fan, XJ. Asymptotically approximate model equations for weakly nonlinear long waves in compressible elastic rods and their comparisons with other simplified model equations. Math Mech Solids 2004; 9: 61-79.

[17] Dai, H-H, and Cai, ZX. An analytical study on the instability phenomena during the phase transitions in a thin strip under uniaxial tension. J Mech Phys Solids 2012; 60: 691-710.

[18] Dai, H-H, and Peng, XC. Elliptic-spline solutions for large localizations in a circular Blatz-Ko cylinder due to geometric softening. SIAM J Appl Math 2012; 70: 181-200.

[19] Dai, H-H, and Wang, F-F. Asymptotic bifurcation solutions for compressions of a clamped nonlinearly elastic rectangle: Transition region and barrelling to a corner-like profile. SIAM J Appl Math 2010; 70: 2673-2692.

[20] Dai, H-H, and Wang, F-F. Analytical solutions for the post-buckling states in the compression of an incompressible hyperelastic layer. Anal Appl 2012; 10: 21-46.

[21] Wang, F-F, and Dai, H-H. Asymptotic bifurcation analysis and post-buckling for uniaxial compression of a thin incompressible hyperelastic rectangle. IMA J Appl Math 2010; 75: 506-524.

[22] Golubitsky, M, and Schaeffer, DG. Imperfect bifurcation in the presence of symmetry. Commun Math Phys 1979; 67: 205-232.

[23] Golubitsky, M, and Schaeffer, DG. Singularities and groups in bifurcation theory, vol. 1 (Applied Mathematical Sciences, vol. 51). New York, NY: Springer, 1985.

[24] Bardi, FC, and Kyriakides, S. Plastic buckling of circular tubes under axial compression. Part I: Experiments. Int J Mech Sci 2006; 48: 830-841.

[25] Willis, AH. Instability in hollow rubber cylinders subjected to axial loads. In: Proceedings of the Seventh International Congress of Applied Mechanics, 1948, 280-296.

[26] Batterman, SC. Plastic buckling of axially compressed cylindrical shells. AIAA J 1965; 3: 316-325.

[27] Lee, LHN. Inelastic buckling of initially imperfect cylindrical shells subject to axial compression. J Aeronaut Sci 1962; 29: 87-95.

[28] Marsolek, J, and Reimerdes, HG. Energy absorption of metallic cylindrical shells with induced non-axisymmetric folding patterns. Int J Impact Eng 2004; 30: 1209-1223.

[29] Ogden, RW. Non-linear elastic deformations. New York, NY: Halsted Press, 1984.

[30] Dai, H-H, and Cai, ZX. Phase transitions in a slender cylinder composed of an incompressible elastic material. I. Asymptotic model equation. Proc R Soc Lond A 2006; 462: 75-95.

[31] Nayfeh, AH. Perturbation methods. New York, NY: Wiley, 1973. 
[32] Fu, YB. Perturbation methods and nonlinear stability analysis. In: Fu, YB, and Ogden, RW (eds) Nonlinear elasticity: Theory and applications. Cambridge: Cambridge University Press, 2011, 345-391.

[33] Doedel, EJ, et al. AUT-07P: Continuation and bifurcation software for ordinary differential equations, August 2007. Available at: http://www.macs.hw.ac. uk/ gabriel/auto07/auto.html

\section{Appendix: Incremental elastic moduli}

For an isotropic hyperelastic material, the strain-energy function $\Phi$ only depends on the three principle stretches of the Lagrangian strain tensor $\mathbf{E}$, that is, $\Phi=\Phi\left(e_{1}, e_{2}, e_{3}\right)$. Denote $\Phi_{j}=\partial \Phi /\left.\partial e_{j}\right|_{e_{1}=e_{2}=e_{3}=0}$ : then in the case where there are no prestresses, $\Phi_{1}, \Phi_{2}$ and $\Phi_{3}$ should vanish.

The non-zero first-order incremental elastic moduli can be written as

$$
\zeta_{1}=A_{1111}^{(1)}=\Phi_{11}, \quad \zeta_{2}=A_{1122}^{(1)}=\Phi_{12}, \quad \zeta_{3}=A_{1212}^{(1)}=\frac{1}{2}\left(\zeta_{1}-\zeta_{2}\right) .
$$

There are only two independent constants among $\zeta_{i}$.

The non-zero second-order incremental elastic moduli can be written as

$$
\begin{aligned}
& \eta_{1}=A_{111111}^{(2)}=\Phi_{111}, \quad \eta_{2}=A_{111122}^{(2)}=\Phi_{112}, \quad \eta_{3}=A_{112233}^{(2)}=\Phi_{123}, \\
& \eta_{4}=A_{111212}^{(2)}=\frac{1}{4}\left(\eta_{1}-\eta_{2}\right), \quad \eta_{5}=A_{331212}^{(2)}=\frac{1}{2}\left(\eta_{2}-\eta_{3}\right), \\
& \eta_{6}=A_{121323}^{(2)}=\frac{1}{8}\left(\eta_{1}-3 \eta_{2}+2 \eta_{3}\right) .
\end{aligned}
$$

There are only three additional non-zero constants among $\eta_{i}$.

The non-zero third-order incremental elastic moduli can be written as

$$
\begin{aligned}
& \kappa_{1}=A_{1111111}^{(3)}=\Phi_{1111}, \quad \kappa_{2}=A_{11111122}^{(3)}=\Phi_{1112}, \quad \kappa_{3}=A_{11112222}^{(3)}=\Phi_{1122}, \\
& \kappa_{4}=A_{11112233}^{(3)}=\Phi_{1123}, \quad \kappa_{5}=A_{11111212}^{(3)}=\frac{1}{6}\left(\kappa_{1}-\kappa_{2}\right), \quad \kappa_{6}=A_{11112323}^{(3)}=\frac{1}{2}\left(\kappa_{3}-\kappa_{4}\right), \\
& \kappa_{7}=A_{11221212}^{(3)}=\frac{1}{12}\left(\kappa_{1}+2 \kappa_{2}-3 \kappa_{3}\right), \quad \kappa_{8}=A_{11221313}^{(3)}=\frac{1}{4}\left(\kappa_{2}-\kappa_{4}\right), \\
& \kappa_{9}=A_{12121212}^{(3)}=\frac{1}{8}\left(\kappa_{1}-4 \kappa_{2}+3 \kappa_{3}\right), \quad \kappa_{10}=A_{12121313}^{(3)}=\frac{1}{24}\left(\kappa_{1}-4 \kappa_{2}+3 \kappa_{3}\right), \\
& \kappa_{11}=A_{11121323}^{(3)}=\frac{1}{24}\left(\kappa_{1}-\kappa_{2}-3 \kappa_{3}+3 \kappa_{4}\right) .
\end{aligned}
$$

There are only four additional non-zero constants among $\kappa_{i}$. 